\title{
THE DUALITY BETWEEN SUBSEMIGROUPS OF LIE GROUPS AND MONOTONE FUNCTIONS
}

\author{
KARL-HERMANN NEEB
}

\begin{abstract}
In this paper we give a characterization of those convex cones $W$ in the Lie algebra $\mathbf{L}(G)$ of a connected Lie group $G$ which are global in $G$, i.e. for which there exists a closed subsemigroup $S$ in $G$ having $W$ as its tangent wedge $\mathbf{L}(S)$. The main result is the Characterization Theorem II.12. We also prove in Corollary II.6 that each germ of a strictly $W$-positive function belongs to a global function if there exists at least one strictly $W$-positive function. We apply the Characterization Theorem to obtain some general conditions for globality and to give a complete description of the global cones in compact Lie algebras.
\end{abstract}

\section{INTRODUCTION}

One of the most essential facts in the theory of Lie groups is that, given a Lie group $G$, there is a one-to-one correspondence between the analytic subgroups of $G$ and the Lie subalgebras of $\mathbf{L}(G)$, the Lie algebra of $G$. We are interested in the corresponding situation in the Lie theory of semigroups.

Semigroups in connection with Lie groups became increasingly important in recent years in such contexts as representation theory $\left(\mathrm{Ol}^{\prime}\right.$ shanskii $[11,12]$, Howe [4]), harmonic analysis (Faraut [3]) and system theory (Kupka [9]). For further references see [7 and 9].

Firstly one has to look for a suitable class of subsemigroups of Lie groups generalizing the analytic subgroups. These are the subsemigroups $S$ of a Lie group $G$ for which the group $G(S):=\left\langle S \cup S^{-1}\right\rangle$ generated by $S$ is an analytic subgroup of $G$. We call these subsemigroups preanalytic. As is described in detail in [7] it is possible to define a tangent wedge for such subsemigroups $S$ of $G$ by

$$
\mathbf{L}(S)=\left\{x \in \mathbf{L}(G): \exp \left(\mathbf{R}^{+} x\right) \subseteq \operatorname{cl}_{G(S)} S\right\},
$$

where the closure has to be understood with respect to the Lie group topology of $G(S)$. This generalizes the notion of a tangent algebra of an analytic subgroup. For a closed subsemigroup $S \subseteq G$ we get

$$
\mathbf{L}(S)=\left\{x \in \mathbf{L}(G): \exp \left(\mathbf{R}^{+} x\right) \subseteq S\right\} .
$$

It is clear that this definition is consistent with the previous one if $S$ is closed and preanalytic. Let us call a closed convex cone $W$ in a finite dimensional

Received by the editors August 21, 1989 and, in revised form, November 20, 1989. This paper has been presented at the AMS meeting in Fayetteville, Arkansas, March 23, 1990.

1980 Mathematics Subject Classification (1985 Revision). Primary 22E15.

The author thanks Professor Dr. Karl H. Hofmann for his support. 
vector space $L$ a wedge and $H(W):=W \cap(-W)$ the edge of the wedge, i.e. the largest vector space contained in $W$. We say that $W$ is pointed if $H(W)=\{0\}$. The suitable generalization of the Lie subalgebras of $\mathbf{L}(G)$ are the Lie wedges. These are the wedges $W \subseteq \mathbf{L}(G)$ with the additional property that

$$
e^{\operatorname{ad} h} W=W \quad \text { for all } h \in H(W) .
$$

Notice that the Lie wedges which are vector spaces are exactly the subalgebras of $\mathbf{L}(G)$. This definition is justified by the fact that for every preanalytic subsemigroup $S$ of a Lie group $G$ the set $\mathbf{L}(S)$ is a Lie wedge [7, V.1.6]. It is also true that, given a Lie wedge $W \subseteq \mathbf{L}(G)$ we find a local subsemigroup $U \subseteq G$ having $W$ as its tangent wedge in some local sense, but the circle is a simple example of a Lie group such that $\mathbf{L}(G)=\mathbf{R}$ contains a Lie wedge $W=\mathbf{R}^{+}$ which is not the tangent wedge of a subsemigroup of $G$. This shows that the correspondence between the subsemigroups of the Lie group $G$ and the Lie wedges in $\mathbf{L}(G)$ is far from being surjective as is true in the group case, where every subalgebra is the tangent object of a subgroup. So it is natural to look for a characterization of those Lie wedges $W \subseteq \mathbf{L}(G)$ which occur as tangent wedges of subsemigroups of $G$. We call these Lie wedges global in $G$. To avoid technical difficulties in our formulations and proofs we often restrict ourselves to subsemigroups $S \subseteq G$ for which $G(S)=G$ and Lie wedges $W \subseteq \mathbf{L}(G)$ which are Lie generating in $\mathbf{L}(G)$, i.e. $\mathbf{L}(G)$ is the smallest subalgebra containing $W$. One knows from [7] that this is no loss in generality.

Our main result on global Lie wedges is Theorem II.12.

Characterization Theorem. A Lie generating Lie wedge $W \subseteq \mathbf{L}(G)$ is global in $G$ if and only if the analytic subgroup corresponding to $H(W)$ is closed in $G$ and we find a function $f \in C^{\infty}(G)$ such that

$$
\left\langle d f(g), d \lambda_{g}(\mathbf{1}) w\right\rangle>0 \quad \text { for all } w \in W \backslash H(W) \text { and } g \in G .
$$

The idea of relating the existence of certain smooth functions to the causality of Lorentz manifolds which is closely related to the globality of Lie wedges can already be found in the book of Hawking and Ellis [5]. See also [12].

The proof of the Characterization Theorem is roughly divided into three main steps. $\S I$ is devoted to the first step, a description of Galois connections from the subsets of $C^{\infty}(G)$ to the subsemigroups of $G$ and to the wedges in $\mathbf{L}(G)$ with respect to set inclusion. We use this in Lemma I.8 to construct for a global Lie generating Lie wedge $W \subseteq \mathbf{L}(G)$ a function $\tilde{f} \in \operatorname{Pos}(W)$ (see Definition I.1) such that

$$
\langle d \tilde{f}(\mathbf{1}), w\rangle>0 \text { for all } w \in W \backslash H(W) .
$$

This result has also been proved in [7, VI] with completely different methods and by doing more hard analysis. Therefore the principal new achievement is the implication that globality of $W$ implies the existence of an $f$ satisfying (1) instead of the weaker condition (2). The second step is to show that, given a function obtained in the first step, we may perturb this function in a neighborhood of 1 (Corollary II.6) to obtain all local germs of strictly $W$-positive functions in a neighborhood of 1 . In the third step we prove that, given a function $\tilde{f}$ satisfying (2), we also find a function $f \in C^{\infty}(G)$ satisfying (1). In the third section of this paper we collect some consequences of the Characterization 
Theorem II.12 which can be obtained with a little extra work. In $\S \mathbf{i V}$ we give some useful methods to construct $W$-positive functions in special cases where their existence is not obvious.

\section{Suitable Galois connections}

Definition I.1. For a subset $\Omega$ of a finite dimensional vector space $L$, we define the dual $\Omega^{*}:=\{\omega \in \widehat{L}:\langle\omega, x\rangle \geq 0$ for all $x \in \Omega\}$. This set is always a wedge in $\widehat{L}$. If $W$ is a wedge we set algint $W=\operatorname{int}_{W-W} W$, where $\operatorname{int}_{W-W} W$ is the interior of $W$ in the space $W-W:=\left\{w-w^{\prime}: w, w^{\prime} \in W\right\}$. According to $[7$, I.2.21] we find that

$$
\text { algint } W^{*}=\{\omega \in \widehat{L}:\langle\omega, w\rangle>0 \text { for all } w \in W \backslash H(W)\} .
$$

Now let $G$ be a Lie group, $L=\mathbf{L}(G)$ its Lie algebra, $S \subseteq G$ a semigroup, $\Omega \subseteq L$, and $M \subseteq C^{\infty}(G)$. For $g \in G$ we write $\lambda_{g}: G \rightarrow G, h \mapsto g h$ for left multiplication by $g$ on $G$. Then we define

(1) $\operatorname{Mon}(S)=\left\{f \in C^{\infty}(G): f(g s) \geq f(g)\right.$ for all $\left.g \in G, s \in S\right\}$. This is the set of smooth functions $f: G \rightarrow \mathbf{R}$ which are monotone with respect to the left invariant order on $G$ defined by $g \leq_{S} g^{\prime}$ if $g^{\prime} \in g S$. We call such functions $S$-monotone.

(2) $\operatorname{Pos}(\Omega):=\operatorname{Mon}\left(\left\langle\exp \mathbf{R}^{+} \Omega\right\rangle\right)$, the set of $\Omega$-positive functions. These are the functions which are increasing along the curves $t \mapsto g \exp (t w)$ for all $w \in \Omega$ and $g \in G$.

(3) $\mathrm{SG}(M)=\{s \in G: f(g s) \geq f(g)$ for all $f \in M, g \in G\}$, the largest subsemigroup $S \subseteq G$ such that all functions $f \in M$ are $S$-monotone.

(4) $\operatorname{ev}(M)=\left\{d f(g) d \lambda_{g}(\mathbf{1}): f \in M, g \in G\right\} \subseteq \widehat{\mathbf{L}(G)}$.

Lemma I.2. For $\Omega \subseteq \mathbf{L}(G), S=\left\langle\exp \mathbf{R}^{+} \Omega\right\rangle$ and $f \in C^{\infty}(G)$ the following assertions are equivalent :

(1) $f \in \operatorname{Mon}(S)$,

(2) $\operatorname{ev}(f) \subseteq \Omega^{*}$.

Proof. We have the following chain of immediate equivalences

$$
\begin{aligned}
f \in \operatorname{Mon}(S) & \Leftrightarrow S \subseteq \operatorname{SG}(f) \Leftrightarrow \exp \left(\mathbf{R}^{+} \Omega\right) \subseteq \operatorname{SG}(f) \\
& \Leftrightarrow f(g \exp (t w)) \geq f(g) \text { for all } g \in G, w \in \Omega, t \in \mathbf{R}^{+} \\
& \Leftrightarrow\left\langle d f(g) d \lambda_{g}(\mathbf{1}), w\right\rangle \geq 0 \text { for all } g \in G, w \in \Omega \\
& \Leftrightarrow \operatorname{ev}(f) \subseteq \Omega^{*} . \quad \square
\end{aligned}
$$

Lemma 1.3. For $M \subseteq C^{\infty}(G)$ we have

$$
\operatorname{ev}(M)^{*}=\mathbf{L}(\operatorname{SG}(M)) \text {. }
$$

Proof. Because $\mathrm{SG}(M)$ is a closed subsemigroup of $G$ we have, according to Lemma I.2, the following equivalences for $x \in \mathbf{L}(G)$

$$
\begin{aligned}
x \in \mathbf{L}(\operatorname{SG}(M)) & \Leftrightarrow \exp \left(\mathbf{R}^{+} x\right) \subseteq \operatorname{SG}(M) \Leftrightarrow M \subseteq \operatorname{Mon}\left(\exp \left(\mathbf{R}^{+} x\right)\right) \\
& \Leftrightarrow \operatorname{ev}(M) \subseteq\left(\mathbf{R}^{+} x\right)^{*} \Leftrightarrow x \in \operatorname{ev}(M)^{*} .
\end{aligned}
$$

Proposition I.4. (1) The mappings $S \mapsto \operatorname{Mon}(S)$ and $M \mapsto \mathrm{SG}(M)$ are inclusion reversing and

$$
M \subseteq \operatorname{Mon}(S) \text { iff } S \subseteq \operatorname{SG}(M) .
$$


Therefore Mon and SG define a Galois connection from the subsemigroups of $G$ to the subsets of $C^{\infty}(G)$.

(2) The mappings $W \mapsto \operatorname{Pos}(W)$ and $M \mapsto \operatorname{ev}(M)^{*}=\mathbf{L}(\operatorname{SG}(M))$ are inclusion reversing and

$$
W \subseteq \operatorname{ev}(M)^{*} \quad \text { iff } \quad M \subseteq \operatorname{Pos}(W) .
$$

Therefore $\mathrm{ev}^{*}$ and Pos define a Galois connection from the wedges in $\mathbf{L}(G)$ to the subsets of $C^{\infty}(G)$.

(3) $S \subseteq \operatorname{SG}(\operatorname{Mon}(S))$ and $\operatorname{Mon}(S)=\operatorname{Mon}(\operatorname{SG}(\operatorname{Mon}(S)))$.

(4) $M \subseteq \operatorname{Mon}(\operatorname{SG}(M))$ and $\operatorname{SG}(M)=\operatorname{SG}(\operatorname{Mon}(\operatorname{SG}(M)))$.

(5) $M \subseteq \operatorname{Pos}\left(\operatorname{ev}(M)^{*}\right)$ and $\operatorname{ev}(M)^{*}=\operatorname{ev}\left(\operatorname{Pos}\left(\operatorname{ev}(M)^{*}\right)\right)^{*}$.

(6) $W \subseteq \operatorname{ev}(\operatorname{Pos}(W))^{*}$ and $\operatorname{Pos}(W)=\operatorname{Pos}\left(\operatorname{ev}(\operatorname{Pos}(W))^{*}\right)$.

Proof. (1) This follows immediately from the definitions.

(2) According to Lemma I.2 we have the following chain of equivalences for a wedge $W \subseteq \mathbf{L}(G)$ and $S=\langle\exp W\rangle$ :

$$
M \subseteq \operatorname{Pos}(W) \Leftrightarrow M \subseteq \operatorname{Mon}(S) \Leftrightarrow \operatorname{ev}(M) \subseteq W^{*} \Leftrightarrow W \subseteq \operatorname{ev}(M)^{*} .
$$

The rest is clear.

(3)-(6) These are standard relations for Galois connections. As an example we show (3). According to (1) $S \subseteq \operatorname{SG}(\operatorname{Mon}(S))$ is equivalent to $\operatorname{Mon}(S) \subseteq$ $\operatorname{Mon}(S)$ which is true. A similar argument shows $\operatorname{Mon}(S) \subseteq \operatorname{Mon}(\operatorname{SG}(\operatorname{Mon}(S)))$ and application of $\operatorname{Mon}$ to $S \subseteq \operatorname{SG}(\operatorname{Mon}(S))$ leads to the reverse inclusion.

We now give a characterization of the fixed points of the mappings SGoMon, Mon $\circ$ SG and $\mathrm{ev}^{*} \circ$ Pos.

Proposition I.5. If $S$ is a closed subsemigroup of a Lie group $G$ then

$$
S=\operatorname{SG}(\operatorname{Mon}(S)) \text {. }
$$

Proof. For $s \in G \backslash S$ we have to find a function $f_{1} \in \operatorname{Mon}(S)$ with $f_{1}(s)<$ $f_{1}(\mathbf{1})$. We choose a left invariant metric $d$ on $G$ and set $f(g):=d(g S, s):=$ $\inf _{s^{\prime} \in S} d\left(g s^{\prime}, s\right)$. Then $f$ is a continuous $S$-monotone function on $G$ with

$$
f(s)=d(s S, s)=d(S, \mathbf{1})=0<f(\mathbf{1})=d(S, s)
$$

because $s \notin S$. For every relatively compact open neighborhood $U$ of $\mathbf{1}$ in $G$ we find a nonnegative function $h_{U} \in C^{\infty}(G)$ with $h(U)=\{1\}, \operatorname{supp}(h) \subseteq U^{2}$ and $\int_{G} h_{U} d m=1$ for a left invariant Haar measure $m$ on $G$. Then

$$
h_{U} * f: g \mapsto \int_{G} h_{U}(x) f\left(x^{-1} g\right) d m(x)
$$

is a $S$-monotone smooth function for every $U$ and the net $h_{U} * f$ converges to $f$, hence we find an $U$ with

$$
h_{U} * f(s)<h_{U} * f(\mathbf{1}) \text {. }
$$

Proposition I.6. The fixed points of $\mathrm{ev}^{*} \circ$ Pos are the tangent wedges of closed subsemigroups of $G$. For a subset $\Omega \subseteq \mathbf{L}(G)$ we have

$$
\operatorname{Pos}(\mathbf{\Omega})=\operatorname{Pos}(\mathbf{L}(\bar{S})) \quad \text { for } S=\left\langle\exp \mathbf{R}^{+} \Omega\right\rangle
$$

and

$$
\operatorname{ev}(\operatorname{Pos}(\Omega))^{*}=\mathbf{L}(\bar{S})=\left\{x \in \mathbf{L}(G): \exp \left(\mathbf{R}^{+} x\right) \subseteq \bar{S}\right\}
$$


Proof. For $\Omega \subseteq \mathbf{L}(G)$ we have

$$
\operatorname{Pos}(\Omega)=\operatorname{Mon}(S)=\operatorname{Mon}(\bar{S}) \subseteq \operatorname{Mon}(\langle\exp \mathbf{L}(\bar{S})\rangle)=\operatorname{Pos}(\mathbf{L}(\bar{S})) \subseteq \operatorname{Pos}(\Omega)
$$

which proves (3). Now let $W=\operatorname{ev}(\operatorname{Pos}(\Omega))^{*}$. From (3) and Lemmas I.3, I.4 and $\mathrm{I} .5$ we get with $M=\operatorname{Pos}(\Omega)$ that

$$
\mathbf{L}(\bar{S})=\mathbf{L}(\operatorname{SG}(\operatorname{Mon}(S)))=\mathbf{L}(\operatorname{SG}(\operatorname{Pos}(\Omega)))=\operatorname{ev}(\operatorname{Pos}(\Omega))^{*}=W
$$

and therefore $W=\mathbf{L}(\bar{S})=\operatorname{ev}\left(\operatorname{Pos}(\mathbf{L}(\bar{S}))^{*}\right.$ by (3). This shows that every fixpoint of $\mathrm{ev}^{*} \circ$ Pos is the tangent wedge of a closed subsemigroup of $G$. Let us assume that $W$ is the tangent wedge of a closed subsemigroup $T$ of $G$ and set $S \stackrel{\text { def }}{=}$ $\overline{\langle\exp W\rangle}$. Then $S \subseteq T$ and $\mathbf{L}(S)=W$. The calculation from above shows that

$$
W=\mathbf{L}(S)=\operatorname{ev}(\operatorname{Pos}(W))^{*}
$$

is a fixed point of $\mathrm{ev}^{*} \circ$ Pos .

Proposition I.7. The fixed points of Mon $\circ$ SG are exactly the convex cones $K$ in $C^{\infty}(G)$ which fulfill the following conditions:

(1) $\mathbf{R} \cdot 1 \subseteq K$, this means that $K$ contains all constant functions.

(2) For all $g \in G$ and $f \in K$ we have ${ }_{g} f:=f \circ \lambda_{g^{-1}} \in K$.

(3) $K$ is closed with respect to the topology of pointwise convergence.

(4) If $f$ is in $K$ and $h$ is nondecreasing and smooth on an open neighborhood of $f(G)$ then $h \circ f \in K$.

(5) For $f, f^{\prime} \in K$ and $h \in C^{\infty}(G)$ with compact support and $h \geq 0$ the functions $h * \min \left(f, f^{\prime}\right)$ and $h * \max \left(f, f^{\prime}\right)$ are contained in $K$.

Proof. Firstly we assume that $K=\operatorname{Mon}(\operatorname{SG}(K))$. Then condition (1) is trivial, (2) follows from

$$
{ }_{g} f(h s)=f\left(g^{-1} h s\right) \geq f\left(g^{-1} h\right)={ }_{g} f(h) \text { for all } g, h \in G \text { and } s \in \operatorname{SG}(K) \text {. }
$$

For $g, s \in G$ the set $F_{g, s}=\left\{f \in C^{\infty}(G): f(g s) \geq f(g)\right\}$ is closed in the topology of pointwise convergence and therefore

$$
K=\bigcap_{\substack{g \in G \\ s \in \mathrm{SG}(K)}} F_{g, s}
$$

is also closed in this topology. This gives (3), and (4) is immediate from $h \circ f(g s)=h(f(g s)) \geq h(f(g))=h \circ f(g)$ for all $f \in K, g \in G, s \in \operatorname{SG}(K)$. Now let $f, f^{\prime} \in K, g \in G$ and $s \in \mathrm{SG}(K)$. We assume that $\min \left(f, f^{\prime}\right)(g s)=$ $f(g s)$. Then

$$
\min \left(f, f^{\prime}\right)(g s)=f(g s) \geq f(g) \geq \min \left(f, f^{\prime}\right)(g)
$$

and after integration we get

$$
\begin{aligned}
h * \max \left(f, f^{\prime}\right)(g s) & =\int_{G} h(x) \max \left(f, f^{\prime}\right)\left(x^{-1} g s\right) d m(x) \\
& \geq \int_{G} h(x) \max \left(f, f^{\prime}\right)\left(x^{-1} g\right) d m(x) \\
& =h * \max \left(f, f^{\prime}\right)(g)
\end{aligned}
$$


because $h$ is a nonnegative function. The fact that $h$ is compactly supported implies that $h * \max \left(f, f^{\prime}\right) \in K \subseteq C^{\infty}(G)$. The proof for max is similar.

Now let $K$ satisfy (1)-(5) and take $\varphi \in \operatorname{Mon}(\operatorname{SG}(K))$. We have to show that $\varphi$ is in $K$. We set $\tilde{\varphi}=\tanh \circ \varphi$ and show that $\tilde{\varphi}$ is in the pointwise closure $M$ of $K \cap B(G)$ in $B(G)$, the space of all bounded functions on $G$. Then we use (4) to get that $\varphi=\operatorname{artanh} \circ \tilde{\varphi} \in K$. If we set $\{\varphi \geq r\}:=\{g \in G: \varphi(g) \geq r\}$ we have $\varphi=\sup _{r \in \mathbf{R}} r \chi_{\{\varphi \geq r\}}$ and therefore $\varphi$ is the pointwise limit of the net of finite sups of the functions $r \chi_{\{\varphi \geq r\}}$. For a set $U \subseteq G$ with $U=U \operatorname{SG}(K)$, $\{\varphi \geq r\}$ for example, we have

$$
\chi_{U}=\sup _{u \in U} \chi_{u \mathrm{SG}(K)}=\sup _{u \in U}\left({ }_{u^{-1}} \chi_{\mathrm{SG}(K)}\right) .
$$

By the same argument as above and (2) it remains to show that $\chi_{\mathrm{SG}(K)} \in M$ and that $M$ is stable under formation of finite sups. If we choose nonnegative functions $h_{n} \in C^{\infty}(G)$ with compact support and total mass 1 such that the supports $\operatorname{supp}\left(h_{n}\right)$ are shrinking to $\{1\}$ we find that

$$
\max \left(f, f^{\prime}\right)=\lim _{n \rightarrow \infty} h_{n} * \max \left(f, f^{\prime}\right) \in M \quad \text { for all } f, f^{\prime} \in K \cap B(G) .
$$

From this it is clear that finite sups and infs of elements of $M$ are in $M$. Using the definition of SG and (1), (2), (4) we see that

$$
\mathrm{SG}(K)=\bigcap_{f \in K}\{f \geq f(\mathbf{1})\}=\bigcap_{\substack{f \in K \cap B(G), f(\mathbf{1})=1 \\ f(G) \subseteq[1 / 2, \infty[}}\{f \geq 1\}
$$

and therefore we get

$$
\chi_{\mathrm{SG}(K)}=\inf _{\substack{f \in K \cap B(G), f(\mathbf{1})=1 \\ f(G) \subseteq[1 / 2, \infty[}} \chi_{\{f \geq 1\}}
$$

for the characteristic functions. But for $\tilde{f}=\min (1, f) \in M$ we have $\{\tilde{f} \geq$ $1\}=\{f \geq 1\}$ so we may restrict our attention to the case where $f(G) \subseteq\left[\frac{1}{2}, 1\right]$. The function $x \mapsto x^{n}$ is smooth and nondecreasing on $] 0, \infty[$ and by (4) and a passage to the limit we have $f^{n} \in M$ for all $n \geq 1$. But now we are done because

$$
\chi_{\{f \geq 1\}}=\lim _{n \rightarrow \infty} f^{n} \in M
$$

The following lemma will be an essential ingredient in the proof of Theorem II.11 (compare [7, VI. 4.10]).

Lemma I.8. Let $S \subseteq G$ be a closed submonoid, then

$$
\mathbf{L}(S)=\operatorname{ev}(\operatorname{Mon}(S))^{*} .
$$

Suppose, in addition, that $\mathbf{L}(S)=W$. Then for every $\omega \in$ algint $W^{*}$ there exists a function $f \in \operatorname{Mon}(S)$ such that $d f(\mathbf{1})=\omega$.

Proof. Using Lemmas I.3 and I.5 we get

$$
\mathbf{L}(S)=\mathbf{L}(\operatorname{SG}(\operatorname{Mon}(S)))=\operatorname{ev}(\operatorname{Mon}(S))^{*} .
$$

Assume that $\mathbf{L}(S)=W$. Then we have $W=\operatorname{ev}(\operatorname{Mon}(S))^{*}$. But $\operatorname{Mon}(S)$ is a convex cone in $C^{\infty}(G)$ which is invariant under left translations (Proposition 
I.7). Hence $\operatorname{ev}(\operatorname{Mon}(S))=\{d f(\mathbf{1}): f \in \operatorname{Mon}(S)\}$ is a convex cone in $\widehat{\mathbf{L}(\boldsymbol{G})}$. For every convex cone $V \subseteq \widehat{\mathbf{L}(G)}$ we have [7, I.1.4, I.2.21]

$$
V^{* *}=\bar{V} \text { and algint } V=\operatorname{int}_{V-V} V=\operatorname{int}_{V-V} \bar{V}=\text { algint } \bar{V} \text {. }
$$

We apply this to $V=\operatorname{ev}(\operatorname{Mon}(S))$ to get

$$
\text { algint } W^{*}=\operatorname{algint} \operatorname{ev}(\operatorname{Mon}(S)) \text {. }
$$

So for every $\omega \in$ algint $W^{*}$ we find a function $f \in \operatorname{Mon}(S)$ with $d f(\mathbf{1})=$ $\omega$.

\section{THE CHARACTERIZATION OF THE GLOBAL LIE WEDGES}

Before we state the main theorem of this section we have to introduce some conventions applying to manifolds with trivial tangent bundle. In the following $G$ is a connected Lie group and $L=\mathbf{L}(G)$ its Lie algebra. On $L$ we introduce a norm such that $\|[x, y]\| \leq\|x\|\|y\|$. We trivialize the tangent bundle of $G$ with the mapping

$$
\Psi: L \times G \rightarrow T(G), \quad(X, g) \mapsto d \lambda_{g}(\mathbf{1}) X .
$$

If $V$ is a finite dimensional vector space and $f: G \rightarrow V$ a differentiable function we define $f^{\prime}: G \rightarrow \operatorname{Hom}(L, V)$ by

$$
\left\langle f^{\prime}(g), X\right\rangle=\left\langle d f(g), d \lambda_{g}(\mathbf{1}) X\right\rangle \text { for all } X \in L .
$$

Successively we get for $f \in C^{n}(G, V)$ functions $f^{(n)}: G \rightarrow \operatorname{Hom}^{n}(L, V)$, where $\operatorname{Hom}^{n}(L, V)$ is recursively defined by $\operatorname{Hom}^{1}(L, V):=\operatorname{Hom}(L, V)$ and $\operatorname{Hom}^{n}(L, V):=\operatorname{Hom}\left(L, \operatorname{Hom}^{n-1}(L, V)\right)$. $\operatorname{Hom}^{n}(L, V)$ is the space of $n$ linear mappings from $L$ to $V$. If $V$ is a normed space we endow the spaces $\operatorname{Hom}^{n}(L, V)$ with the natural operator norms. We write $B M^{+}(G)$ for the space of all nonnegative bounded regular Borel measures on $G$.

Lemma II.1. Let $C \neq\{0\}$ be a pointed wedge in a finite dimensional vector space $L$ and $K \subseteq \operatorname{int} C^{*}$ a compact subset. Then we find a pointed wedge $C^{\prime} \subseteq L$ such that

(1) $K \subseteq \operatorname{int}\left(C^{\prime}\right)^{*}$ and

(2) $C^{\prime}$ surrounds $C$, i.e. $C \backslash\{0\} \subseteq \operatorname{int} C^{\prime}$.

Proof. We choose a compact convex subset $K^{\prime} \subseteq \operatorname{int} C^{*}$ which contains $K$ in its interior. This is possible because the convex closure of $K$ is also a compact convex subset of the interior of $C^{*}$. But now $0 \notin K^{\prime} \subseteq \operatorname{int} C^{*}$ because otherwise we would have $\mathbf{R}^{+} K^{\prime}=\widehat{L} \subseteq C^{*}$ and therefore $C=\{0\}$ which was excluded. Then $\mathbf{R}^{+} K^{\prime}$ is a pointed generating wedge in $\widehat{L}$. We set $C^{\prime}:=$ $\left(\mathbf{R}^{+} K^{\prime}\right)^{*}=K^{\prime *}$. Then (1) is immediate from the construction. Suppose that $C^{\prime}$ does not surround $C$. Then we find a functional $\omega \in K^{\prime} \subseteq C^{\prime *}$ and a point $x \in C \cap \partial C^{\prime}$ such that $\langle\omega, x\rangle=0$. This is a contradiction to $K^{\prime} \subseteq \operatorname{int} C^{*}$.

For the proof of Proposition II.3 we need the following lemma.

Lemma II.2. Let $U \subseteq \mathbf{R}^{n}$ be an open neighborhood of $0,0 \neq v \in \mathbf{R}^{n}$ and $V$ be a convex neighborhood of 0 which contains $v$. Then we find a function $g \in C^{\infty}\left(\mathbf{R}^{n}\right)$ such that 
(1) $\operatorname{supp}(g) \subseteq U$ is compact,

(2) $g(0)=0, \nabla g=v$ on a neighborhood of 0 , and

(3) $\nabla g(x) \in V$ for all $x \in U$.

Proof. Using a suitable base in $\mathbf{R}^{n}$ and after shrinking $U$ and $V$ we may assume that

$$
U=\left\{x \in \mathbf{R}^{n}:\|x\|_{\infty}<1\right\}, \quad v=(\lambda, 0, \ldots, 0), \quad \lambda>0,
$$

and

$$
V=\left\{x \in \mathbf{R}^{n}:-\delta \leq x_{1} \leq \lambda+\delta,\left|x_{j}\right| \leq \delta \text { for all } j>1\right\} \text { with } 0<\delta<1 .
$$

The special size of $V$ is possible because $V$ contains a $\delta$-ball around 0 and $v$ in the sup-norm $\|\cdot\|_{\infty}$ and therefore the convex hull of their union.

Now let $\gamma \in C^{\infty}(\mathbf{R})$ with $\left.\operatorname{supp}(\gamma) \subseteq\right]-1,1[, \gamma=1$ on a neighborhood of 0 and $M:=\sup _{x \in \mathbf{R}}\left|\gamma^{\prime}(x)\right| \geq 1$. We set $\varepsilon:=\min \left\{\frac{1}{2}, \frac{\delta}{(\lambda+\delta)}, \frac{\delta}{\lambda M}\right\}$ and choose two functions $\beta_{1}, \beta_{2} \in C^{\infty}(\mathbf{R})$ such that

(i) $\left.\operatorname{supp}\left(\beta_{1}\right) \subseteq\right]-\varepsilon, \varepsilon\left[, \beta_{1}\right.$ is symmetric and $\left.\operatorname{supp}\left(\beta_{2}\right) \subseteq\right]-1,-\varepsilon[$,

(ii) $0 \leq \beta_{1}(t) \leq \lambda,-\delta \leq \beta_{2}(t) \leq 0$ for all $t \in \mathbf{R}$,

(iii) $\beta_{1}=\lambda$ on a neighborhood of 0 , and

(iv) $\int_{\mathbf{R}} \beta_{1}(x) d x=\lambda \varepsilon, \int_{\mathbf{R}} \beta_{2}(x) d x=-\lambda \varepsilon / 2$.

To show that this is possible we have to check that (iv) is fulfillable. For $\beta_{1}$ this is clear and for $\beta_{2}$ it follows directly from $\delta(1-\varepsilon) \geq \varepsilon \lambda$ which is the same as $\varepsilon \leq \delta /(\lambda+\delta)$. Now we define

$$
\beta(t)= \begin{cases}\beta_{2}(t), & \text { if } t \leq-\varepsilon \\ \beta_{1}(t), & \text { if }-\varepsilon \leq t \leq \varepsilon ; \\ \beta_{2}(-t), & \text { if } t \geq \varepsilon .\end{cases}
$$

The support of $\beta$ is contained in ] $-1,1[$ and the conditions from above imply that $\beta=\lambda$ on a neighborhood of $0,-\delta \leq \beta(t) \leq \lambda$ and $\int_{\mathbf{R}} \beta(x) d x=$ $\lambda(-\varepsilon / 2+\varepsilon-\varepsilon / 2)=0$. The function $\alpha$ defined by $\alpha(t)=\int_{-\infty}^{t} \beta(s) d s$ has compact support in ] $-1,1$ [, is nonincreasing on ] $-1,-\varepsilon[$ and $] \varepsilon, 1$ [, nondecreasing on $]-\varepsilon, \varepsilon[$ and has the extreme values

$$
\alpha(-\varepsilon)=\int_{\mathbf{R}} \beta_{2}(t) d t=-\frac{\lambda \varepsilon}{2}, \quad \alpha(\varepsilon)=0-\int_{\mathbf{R}} \beta_{2}(-t) d t=\frac{\lambda \varepsilon}{2} .
$$

The symmetry of $\beta$ implies that $\alpha(0)=0$. Consequently $\max _{x \in \mathbf{R}}|\alpha(x)| \leq$ $\varepsilon \lambda / 2,-\delta \leq \alpha^{\prime}=\beta \leq \lambda$ and $\alpha^{\prime}=\lambda$ on a neighborhood of 0 . Now we have all bricks to build the desired function. We set

$$
g(x)=g\left(x_{1}, x_{2}, \ldots, x_{n}\right)=\alpha\left(x_{1}\right) \prod_{i=2}^{n} \gamma\left(x_{i}\right) .
$$

This function has the desired properties:

(i) $\operatorname{supp}(g) \subseteq\left\{x:\left\|x_{i}\right\|_{\infty}<1\right.$, for all $\left.i=1, \ldots, n\right\}$.

(ii) $g(0)=0$ and $\nabla g=(\lambda, 0, \ldots, 0)$ on a neighborhood of 0 , and

(iii) $\nabla g(x) \in[-\delta, \lambda] \times[-\delta, \delta]^{n-1} \subseteq V$, because we have

$$
\nabla g(x)=\left(\alpha^{\prime}\left(x_{1}\right) \prod_{i>1} \gamma\left(x_{i}\right), \ldots, \alpha\left(x_{1}\right) \gamma^{\prime}\left(x_{j}\right) \prod_{1<i \neq j} \gamma\left(x_{i}\right), \ldots\right)
$$


where

$$
-\delta \leq \alpha^{\prime}\left(x_{1}\right) \prod_{i>1} \gamma\left(x_{i}\right) \leq \lambda \prod_{i>1} \gamma\left(x_{i}\right) \leq \lambda
$$

and

$$
\left|\alpha\left(x_{1}\right) \gamma^{\prime}\left(x_{j}\right) \prod_{1<i \neq j} \gamma\left(x_{i}\right)\right| \leq \max _{x \in \mathbf{R}}|\alpha(x)| \max _{x \in \mathbf{R}}\left|\gamma^{\prime}(x)\right| \leq \varepsilon \frac{\lambda}{2} M<\delta .
$$

This completes the proof of the lemma.

Proposition II.3 (The Perturbation Principle). Let $M$ be a differentiable $n$ dimensional manifold with trivial tangent bundle, $p \in M, T(M)=T_{p}(M) \times M$, $W \subseteq T_{p}(M)$ a pointed wedge and $\theta: x \mapsto W \times\{x\} \subseteq T_{x}(M)$ the associated constant wedge field. Suppose that $f \in C^{\infty}(M)$ such that $d f(p) \in \operatorname{int} \theta(p)^{*}$ and $h \in C^{\infty}(M)$ such that $d h(p) \neq 0, h(p)=0$ and $d f(p)+d h(p) \in \operatorname{int} \theta(p)^{*}$ Then we find an open neighborhood $U$ of $p$ and a function $g \in C^{\infty}(G)$ such that

(1) $\operatorname{supp}(g) \subseteq U$,

(2) $h=g$ on a neighborhood of $p$ and

(3) $d f(x)+d g(x) \in$ int $\theta(x)^{*}$ for all $x \in U$.

Proof. Firstly we choose a neighborhood $U_{1}$ of $p$ in $M$ such that $d f(x)$ and $d f(x)+d h(x) \in$ int $\theta(x)^{*}$ for all $x \in U_{1}$ and that we find functions $\varphi_{2}, \ldots, \varphi_{n} \in C^{\infty}\left(U_{1}\right)$ such that $\varphi:=\left(h, \varphi_{2}, \ldots, \varphi_{n}\right): U_{1} \rightarrow \varphi\left(U_{1}\right)$ is a diffeomorphism, i.e. a chart for $M$, with $\varphi(p)=0$. This is possible because $d h(p) \neq 0$. Using Lemma II.1 we find a pointed cone $W^{\prime} \subseteq T_{p}(M)$ such that $d f(p), d f(p)+d h(p) \in$ int $W^{\prime *}$ and $W^{\prime}$ surrounds $W$.

Now we transport our problem to $\mathbf{R}^{n}$. Set $C:=d \varphi(p) W, C:=d \varphi(p) W^{\prime}$, $\tilde{\theta}(y)=d \varphi\left(\varphi^{-1}(y)\right) \theta\left(\varphi^{-1}(y)\right)$ for $y \in \varphi\left(U_{1}\right), \tilde{f}=f \circ \varphi^{-1}$ and $\tilde{h}=h \circ \varphi^{-1}$ on $\varphi\left(U_{1}\right)$. Then $\tilde{h}(x)=x_{1}$ is the first coordinate function on $\varphi\left(U_{1}\right), \nabla \tilde{h}(y)=$ $e_{1}:=(1,0, \ldots, 0), C^{\prime}$ surrounds $C$ and $\nabla \tilde{f}(0), \nabla \tilde{f}(0)+e_{1} \in \operatorname{int} C^{* *}$.

We fix a compact base $B_{W}$ of $W$. The mapping

$$
(v, x) \mapsto d \varphi(x) v, \quad T_{p}(M) \times U_{1} \rightarrow \mathbf{R}^{n}
$$

is continuous and $d \varphi(p) B_{W} \subseteq$ int $C^{\prime}$. We find an open neighborhood $U_{2} \subseteq U_{1}$ of $p$ such that $d \varphi(x) B_{W} \subseteq$ int $C^{\prime}$ for all $x \in U_{2}$ and therefore $C^{\prime}$ surrounds $\tilde{\theta}(y)$ for all $y \in \varphi\left(U_{2}\right)$. In addition we may assume that $d \tilde{f}(y) \in \operatorname{int} C^{\prime *}$ for all $y \in U_{2}$. Now our task is to find a function $\tilde{g} \in C^{\infty}\left(\mathbf{R}^{n}\right)$ such that

(1) $\operatorname{supp}(\tilde{g}) \subseteq \varphi\left(U_{2}\right)$ is compact,

(2) $\tilde{g}(0)=0$ and $\nabla \tilde{g}=e_{1}$ on a neighborhood of 0 , and

(3) $\nabla \tilde{f}(x)+\nabla \tilde{g}(x) \in \operatorname{int} C^{*}$ for all $x \in \varphi\left(U_{2}\right)$.

Then we set $U:=U_{2}$ and

$$
g(x)= \begin{cases}\tilde{g}(\varphi(x)), & \text { for } x \in U_{2} \\ 0, & \text { for } x \notin U_{2}\end{cases}
$$

This clearly defines a smooth function on $M$ because $\tilde{g}$ has compact support in the open set $\varphi\left(U_{2}\right)$. The first two conditions from above are satisfied. We have to check the last one. For $x \in U=U_{2}$ we have

$$
\begin{aligned}
d f(x)+d g(x) & =d \widehat{\varphi(x)}(\nabla \tilde{f}(\varphi(x))+\nabla \tilde{g}(\varphi(x))) \in d \widehat{\varphi(x)} \text { int } C^{*} \\
& \left.\subseteq d \widehat{\varphi(x)} \operatorname{int} \tilde{\theta}(\varphi(x))^{*}=\operatorname{int} \widehat{d \widehat{\varphi(x)}} d \widehat{\varphi(x)}\right)^{-1} \theta(x)^{*}=\operatorname{int} \theta(x)^{*} .
\end{aligned}
$$


To apply Lemma II.2 we have to reformulate the problem. We find a compact neighborhood $U_{3} \subseteq \varphi\left(U_{2}\right)$ of 0 such that $\nabla \tilde{f}(x)+e_{1} \in \operatorname{int} C^{\prime *}$ for all $x \in U_{3}$. Let $K=\operatorname{conv}\left\{\nabla \tilde{f}(x): x \in U_{3}\right\}$. Then $K \cup\left(e_{1}+K\right) \subseteq$ int $C^{\prime *}$ and the set $V=\left\{x: K+x \subseteq\right.$ int $\left.C^{*}\right\}$ is an open convex neighborhood of 0 and $e_{1}$ such that $K+V \subseteq$ int $C^{\prime *}$. Now it is readily verified that it is enough to find $\tilde{g} \in C^{\infty}\left(\mathbf{R}^{n}\right)$ such that

(1) $\operatorname{supp}(\tilde{g}) \subseteq \operatorname{int} U_{3}$ is compact,

(2) $\tilde{g}(0)=0, \nabla \tilde{g}=e_{1}$ on a neighborhood of 0 , and

(3) $\nabla \tilde{g}(x) \in V$ for all $x \in U_{3}$.

This is exactly what we get from Lemma II.2 and the proposition is proved.

Proposition II.4. Let $M=G / H$ be a homogeneous $G$-space and $W \subseteq \mathbf{L}(G)$ a Lie wedge with $\mathbf{L}(H)=H(W)$ which is invariant under $\operatorname{Ad}(H)$. Then every local cross section $\sigma: U \rightarrow G$ with $U \subseteq G / H$ of the projection $\pi: G \rightarrow G / H$ provides a trivialization of the tangent bundle $T(U)$ such that the wedge field $\theta(\pi(x))=d \pi(x) d \lambda_{x}(\mathbf{1}) W$ is constant. This trivialization is defined by

$$
\begin{gathered}
\Psi: T(U) \rightarrow U \times T_{\pi(\mathbf{1})}(G / H), \\
\Psi\left(X_{p}\right)=\left(p, d \pi(\mathbf{1}) d \lambda_{\sigma(p)}(\mathbf{1})^{-1} d \sigma(p) X_{p}\right) .
\end{gathered}
$$

Proof. Firstly the mapping $\Psi$ is an isomorphism of vector bundles [2] which is linear on the fibres and fixes the base $U$. We show that it is one-to-one on each fibre. Let

$$
d \pi(\mathbf{1}) d \lambda_{\sigma(p)}(\mathbf{1})^{-1} d \sigma(p) X_{p}=0 .
$$

Then $d \sigma(p) X_{p} \in d \lambda_{\sigma(p)}(\mathbf{1}) \mathbf{L}(H)=\operatorname{ker} d \pi(\sigma(p))$ and therefore

$$
d \pi(\sigma(p)) d \sigma(p) X_{p}=d(\pi \circ \sigma)(p) X_{p}=X_{p}=0 .
$$

Consequently $\Psi$ is injective on each fibre and therefore one-to-one. Define $\mu_{g}: G / H \rightarrow G / H$ for $g \in G$ by $\mu_{g} \circ \pi=\pi \circ \lambda_{g}$. Taking differentials we have

$$
d \mu_{g}\left(\pi\left(g^{\prime}\right)\right) \circ d \pi\left(g^{\prime}\right)=d \pi\left(g g^{\prime}\right) \circ d \lambda_{g}\left(g^{\prime}\right) \text { for } g, g^{\prime} \in G \text {. }
$$

For the wedge field $\theta$ at $p \in U$ we have

$$
\begin{aligned}
d \pi(\mathbf{1}) & d \lambda_{\sigma(p)}(\mathbf{1})^{-1} d \sigma(p) \theta(p)=d \pi(\mathbf{1}) d \lambda_{\sigma(p)^{-1}}(\sigma(p)) d \sigma(p) \theta(p) \\
& =d \mu_{\sigma(p)^{-1}}(p) d \pi(\sigma(p)) d \sigma(p) \theta(p)=d \mu_{\sigma(p)^{-1}}(p) d(\pi \circ \sigma)(p) \theta(p) \\
& =d \mu_{\sigma(p)^{-1}}(p) \theta(p)=d \mu_{\sigma(p)^{-1}}(p) d \pi(\sigma(p)) d \lambda_{\sigma(p)}(\mathbf{1}) W \\
& =d \pi(\mathbf{1}) d \lambda_{\sigma(p)^{-1}}(\sigma(p)) d \lambda_{\sigma(p)}(\mathbf{1}) W=d \pi(\mathbf{1}) W=\theta(\pi(\mathbf{1})) .
\end{aligned}
$$

Therefore the wedge field $\theta$ is constant in this trivialization.

Lemma II.5. Let $G$ be a connected Lie group, $H \subseteq G$ a closed subgroup, $\pi: G \rightarrow G / H$ the projection, $W \subseteq \mathbf{L}(G)$ a Lie wedge such that $\mathbf{L}(H) \subseteq H(W)$, $\theta(\pi(x))=d \pi(x) d \lambda_{x}(\mathbf{1}) W$ and $U=U H$ a neighborhood of $H$ in $G$. Then the mapping $\Phi: C^{\infty}(\pi(U)) \rightarrow C^{\infty}(U), \tilde{f} \mapsto f=\tilde{f} \circ \pi$ is one-to-one from

$$
\operatorname{Pos}_{G / H, U}(W)=\left\{\tilde{f} \in C^{\infty}(\pi(U)): d \tilde{f}(\pi(x)) \in \theta(\pi(x))^{*} \text { for all } x \in U\right\}
$$

to

$$
\operatorname{Pos}_{U}(W)=\left\{f \in C^{\infty}(U): f^{\prime}(x) \in W^{*} \text { for all } x \in U\right\}
$$


$\Phi$ is surjective if $H$ is connected. The following conditions are equivalent for $f \in \operatorname{Pos}_{U}(W)$ and $x \in U$

(1) $f^{\prime}(x) \in$ algint $W^{*}$ and

(2) $d \tilde{f}(\pi(x)) \in$ algint $\theta(\pi(x))^{*}$.

Proof. Let $\tilde{f} \in \operatorname{Pos}_{G / H, U}(W), x \in U$ and $v=d \pi(x) w$ with $w \in d \lambda_{x}(\mathbf{1}) W$. Then

$$
\langle d \tilde{f}(\pi(x)), v\rangle=\langle d \tilde{f}(\pi(x)), d \pi(x) w\rangle=\langle d f(x), w\rangle \geq 0,
$$

hence $f \in \operatorname{Pos}_{U}(W)$ and

$$
\Phi\left(\operatorname{Pos}_{G / H, U}(W)\right) \subseteq \operatorname{Pos}_{U}(W) .
$$

The function $\tilde{f}$ is clearly uniquely determined by $f$ and therefore $\Phi$ is one-toone from $\operatorname{Pos}_{G / H, U}(W)$ to $\operatorname{Pos}_{U}(W)$. The equivalence of (1) and (2) follows from the following chain of equivalences (Definition I.1)

$$
\begin{aligned}
f^{\prime}(g) \in \text { algint } W^{*} & \Leftrightarrow\left\langle d f(x), d \lambda_{x}(\mathbf{1}) w\right\rangle>0 \text { for } w \in W \backslash H(W) \\
& \Leftrightarrow\left\langle d \tilde{f}(\pi(x)), d \pi(x) d \lambda_{x}(\mathbf{1}) w\right\rangle>0 \text { for } w \in W \backslash H(W) \\
& \Leftrightarrow\langle d \tilde{f}(\pi(x)), v\rangle>0 \text { for } v \in \theta(\pi(x)) \backslash H(\theta(\pi(x))) \\
& \Leftrightarrow d \tilde{f}(\pi(x)) \in \text { algint } \theta(\pi(x))^{*} .
\end{aligned}
$$

Assume, in addition, that $H$ is connected and $f \in \operatorname{Pos}_{U}(W)$. Then $f^{\prime}(x) \mathbf{L}(H)$ $\subseteq d f(x) d \lambda_{x}(\mathbf{1}) H(W)=\{0\}$ for all $x \in U$ and, because $H$ is connected, $f$ is constant on the cosets $x H$ for $x \in U$. Therefore we find a function $\tilde{f} \in C^{\infty}(\pi(U))$ such that $\tilde{f} \circ \pi=f$ on $U$. This shows that $\Phi$ is surjective.

Corollary II.6. Let $G$ be a connected Lie group, $H \subseteq G$ a closed connected subgroup, $W \subseteq \mathbf{L}(G)$ a Lie wedge such that $\mathbf{L}(H)=H(W)$ and $U=U H$ an open neighborhood of $H$ in $G$. Suppose that $f \in \operatorname{Pos}(W)$ such that $f^{\prime}(\mathbf{1}) \in$ algint $W^{*}$, then for every function $f_{1} \in \operatorname{Pos}_{U}(W)$ with $f_{1}^{\prime}(x) \in$ algint $W^{*}$ for $x \in U$, we find a function $f_{2} \in \operatorname{Pos}(W)$ which agrees with $f_{1}$ on a small neighborhood of $\mathbf{1}$.

Proof. Let $\pi: G \rightarrow G / H$ be the quotient mapping and $L=E \oplus H(W)$ with a vector space complement $E$ for $H(W)$ in $L$. By the Units Neighborhood Theorem [7, V.2.4.] we find a neighborhood $C$ of 0 in $E$ such that the mapping $m: C \times H \rightarrow \exp (C) H,(c, h) \mapsto \exp (c) h$ is a diffeomorphism onto an open neighborhood of $H$ in $G$. Let $U_{1}:=\pi(\exp C)=\pi(\exp (C) H)$. Then this diffeomorphism provides us with a local cross section $\sigma: U_{1} \rightarrow G, \pi(\exp c) \mapsto$ $\exp (c)$ which is smooth because it is defined by factorization of the smooth mapping $\exp (c) h \mapsto \exp (c), \exp (C) H \rightarrow \exp (C)$ which is constant on the cosets of $H$. We trivialize $T(U)$ as in Proposition II.4 and get a constant wedge field $\theta$ in this trivialization.

We establish that we may assume that $d f_{1}(\mathbf{1}) \neq d f(\mathbf{1})$ because we can multiply $f$ with a positive constant. By adding a constant we may also assume that $f_{1}(\mathbf{1})=f(\mathbf{1})$. Using Lemma II.5 we transport our problem to $G / H$. We find functions $\tilde{f}$ and $\tilde{f}_{1}$ such that $\tilde{f} \circ \pi=f$ and $\tilde{f}_{1} \circ \pi=f_{1}$ on $U$. Let $p=\pi(1)$. Then we have in addition that $d \tilde{f}(p) \in \operatorname{int} \theta(p)^{*}$ and $d \tilde{f}_{1}(p) \in \operatorname{int} \theta(p)^{*}$. For $\tilde{h}=\tilde{f}_{1}-\tilde{f}$ on $\pi(U)$ we have $h(p)=0, d h(p) \neq 0$ and $d \tilde{f}(p)+d \tilde{h}(p)=d \tilde{f}_{1}(p) \in \operatorname{int} \theta(p)^{*}$. Using Proposition II.4 we apply 
Proposition II.3 with $M=\pi(U)$ and find a function $\tilde{g} \in C^{\infty}(G / H)$ and a compact neighborhood $U_{1}$ of $p$ in $\varphi(U)$ such that

(1) $\operatorname{supp}(\tilde{g}) \subseteq U_{1} \subseteq \pi(U)$,

(2) $\tilde{h}=\tilde{g}$ on a neighborhood of $p$, and

(3) $d \tilde{f}(x)+d \tilde{g}(x) \in \operatorname{int} \theta(x)$ for all $x \in U_{1}$.

This implies that $\tilde{f}+\tilde{g} \in \operatorname{Pos}_{G / H, U}(W)$ and that $\tilde{f}+\tilde{g}$ agrees with $\tilde{f}+\tilde{h}=\tilde{f}_{1}$ on a neighborhood of $p$. Now $f_{2}:=f+\tilde{g} \circ \pi$ is the desired function (Lemma II.5).

Lemma II.7. Let $h \in C(G)$ and $U \subseteq G$ be compact. Then the function

$$
h_{U}: G \rightarrow \mathbf{R}, \quad g \mapsto \max _{u \in U} h\left(g^{-1} u\right)
$$

is continuous.

Proof. Let $g \in G, \varepsilon>0$, and $N$ be a symmetric neighborhood of 1 in $G$. Then $h$ is right uniformly continuous on the compact set $N^{-1} U$. So we find another symmetric neighborhood $V \subseteq N$ of 1 such that $|h(y x)-h(x)| \leq \varepsilon$ for $x \in g^{-1} U$ and $y \in V$. For $y \in V$ we find $x_{0}, x_{1} \in U$ such that $h_{U}(g y)=$ $h\left(y^{-1} g^{-1} x_{0}\right)$ and $h_{U}(g)=h\left(g^{-1} x_{1}\right)$. But then we have

$$
h_{U}(g y)=h\left(y^{-1} g^{-1} x_{0}\right) \leq h\left(g^{-1} x_{0}\right)+\varepsilon \leq h_{U}(g)+\varepsilon
$$

and

$$
h_{U}(g)=h\left(g^{-1} x_{1}\right) \leq h\left(y^{-1} g^{-1} x_{1}\right)+\varepsilon \leq h_{U}(g y)+\varepsilon .
$$

Consequently $h_{U}$ is continuous in $g$.

Definition II.8. Let $V$ be a finite dimensional normed space and $f \in C(G, V)$. For a compact subset $N \subseteq G$ we define

$$
f_{N}: G \rightarrow \mathbf{R}^{+}, \quad x \mapsto \max _{n \in N}\left\|f\left(x^{-1} n\right)\right\| .
$$

By the preceding Lemma II.7 this function is continuous on $G$. For $\mu \in$ $B M^{+}(G)$ we call $f$ strongly $\mu$-integrable if $f_{N}$ is $\mu$-integrable for all compact subsets $N \subseteq G$. It is clear that every bounded function is strongly $\mu$-integrable and every continuous function is strongly $\mu$-integrable if $\mu$ has compact support because $f_{N}$ is bounded on $\operatorname{supp} \mu$.

Proposition II.9. Let $V$ be a finite dimensional vector space, $\mu \in B M^{+}(G)$ and $\chi \in C(G, V)$ strongly $\mu$-integrable. Then

(1) $\mu * \chi=\int_{g} \chi d \mu(g) \in C(G, V)$.

(2) Suppose that $\chi \in C^{1}(G, V)$ and $\chi^{\prime}$ is strongly $\mu$-integrable, then $\mu * \chi \in$ $C^{1}(G, V)$ and

$$
(\mu * \chi)^{\prime}=\mu * \chi^{\prime}
$$

in the left trivialization of $G$.

(3) Let $\chi$ be as in (2), $V=\mathbf{R}, W \subseteq \mathbf{L}(G)$ be a wedge, $\operatorname{ev}(\chi) \subseteq W^{*}$ and $x_{0} \in G$. Then $\operatorname{ev}(\mu * \chi) \subseteq W^{*}$ and if $\chi^{\prime}(g) \in$ algint $W^{*}$ and $x_{0} \in \operatorname{supp}(\mu)$ we have

$$
\mu * \chi^{\prime}\left(x_{0} g\right) \in \text { algint } W^{*} \text {. }
$$

Proof. (1) Let $g \in G$ and $\varepsilon>0$. Firstly we choose a compact neighborhood $N$ of $g$. Because $\chi$ is strongly $\mu$-integrable we find a compact subset $K \subseteq G$ 
such that $\int_{G \backslash K} \chi_{N}(x) d \mu(x) \leq \frac{\varepsilon}{4}$. The function $\chi$ is left uniformly continuous on the compact set $K^{-1} N$ and therefore we find a neighborhood $U \subseteq g^{-1} N$ of 1 such that

$$
\|\chi(y h)-\chi(y)\|<\frac{\varepsilon}{2 \mu(G)} \quad \text { for } h \in U, y \in K^{-1} N .
$$

This implies for $h \in U$ that

$$
\begin{aligned}
\| \mu & * \chi(g h)-\mu * \chi(g) \| \\
& \leq\left\|\int_{K} \chi\left(x^{-1} g h\right)-\chi\left(x^{-1} g\right) d \mu(x)\right\|+\left\|\int_{G \backslash K} \chi\left(x^{-1} g h\right)-\chi\left(x^{-1} g\right) d \mu(x)\right\| \\
& \leq\left\|\int_{K} \chi\left(x^{-1} g h\right)-\chi\left(x^{-1} g\right) d \mu(x)\right\|+\int_{G \backslash K}\left\|\chi\left(x^{-1} g h\right)\right\|+\left\|\chi\left(x^{-1} g\right)\right\| d \mu(x) \\
& \leq \frac{\varepsilon \mu(K)}{2 \mu(G)}+2 \frac{\varepsilon}{4} \leq \varepsilon .
\end{aligned}
$$

This shows that $\mu * \chi$ is continuous.

(2) From (1) we get the continuity of $\mu * \chi^{\prime}$. Let $g \in G, \varepsilon>0$ and $y \in L$. For $\chi^{\prime}$ we choose the sets $N, U$ and $K$ as in (1) for $\chi$. In addition we find a $T>0$ such that $\exp ([-T, T] y) \subseteq U$. This leads to

$$
\begin{aligned}
\| \frac{1}{t}( & * \chi(g \exp (t y))-\mu * \chi(g))-\left\langle\mu * \chi^{\prime}(g), y\right\rangle \| \\
& =\left\|\int_{G} \frac{1}{t}\left(\chi\left(x^{-1} g \exp (t y)\right)-\chi\left(x^{-1} g\right)\right)-\left\langle\chi^{\prime}\left(x^{-1} g\right), y\right\rangle d \mu(x)\right\| \\
& =\left\|\int_{G} \frac{1}{t} \int_{0}^{t} \frac{d}{d s} \chi\left(x^{-1} g \exp (s y)\right) d s-\left\langle\chi^{\prime}\left(x^{-1} g\right), y\right\rangle d \mu(x)\right\| \\
& =\| \int_{G} \frac{1}{t} \int_{0}^{t}\left\langle d \chi\left(x^{-1} g \exp (s y)\right) d \lambda_{x^{-1}} g \exp (s y)(\mathbf{1}), y\right\rangle d s \\
& =\left\|\int_{G} \frac{1}{t} \int_{0}^{t}\left\langle\chi^{\prime}\left(x^{-1} g \exp (s y)\right)-\chi^{\prime}\left(x^{-1} g\right), y\right\rangle d s d \mu(x)\right\| \\
& =\left\|\frac{1}{t} \int_{0}^{t} \int_{G}\left\langle\chi^{\prime}\left(x^{-1} g \exp (s y)\right)-\chi^{\prime}\left(x^{-1} g\right), y\right\rangle d \mu(x) d s\right\|
\end{aligned}
$$

This follows from Fubini's Theorem because all measures involved are finite and

$$
\begin{aligned}
& \int_{0}^{t} \int_{G}\left\|\chi^{\prime}\left(x^{-1} g \exp (s y)\right)\right\| d \mu(x) d s \\
& \quad \leq \int_{0}^{t}\left(\frac{\varepsilon}{4}+\int_{K} \chi_{N}(x) d \mu(x)\right) d t<\infty .
\end{aligned}
$$


We go on with our computation:

$$
\begin{aligned}
& \left\|\frac{1}{t} \int_{0}^{t} \int_{G}\left\langle\chi^{\prime}\left(x^{-1} g \exp (s y)\right)-\chi^{\prime}\left(x^{-1} g\right), y\right\rangle d \mu(x) d s\right\| \\
& \leq \frac{1}{t} \int_{0}^{t} \int_{G}\left\|\chi^{\prime}\left(x^{-1} g \exp (s y)\right)-\chi^{\prime}\left(x^{-1} g\right)\right\| d \mu(x) d s\|y\| \\
& =\frac{1}{t} \int_{0}^{t}\left(\int_{K}\left\|\chi^{\prime}\left(x^{-1} g \exp (s y)\right)-\chi^{\prime}\left(x^{-1} g\right)\right\| d \mu(x)\right. \\
& \left.\quad+\int_{G \backslash K}\left\|\chi^{\prime}\left(x^{-1} g \exp (s y)\right)-\chi^{\prime}\left(x^{-1} g\right)\right\| d \mu(x)\right) d s\|y\| \\
& =\frac{1}{t} \int_{0}^{t}\left(\frac{\varepsilon \mu(K)}{2 \mu(G)}+\frac{2 \varepsilon}{4}\right) d s\|y\| \leq\left(\frac{\varepsilon}{2}+\frac{\varepsilon}{2}\right)\|y\|=\varepsilon\|y\| .
\end{aligned}
$$

This completes the proof of $(\mu * \chi)^{\prime}=\mu * \chi^{\prime}$.

(3) For $w \in W$ we have

$$
\left\langle\mu * \chi^{\prime}(g), w\right\rangle=\int_{G}\left\langle\chi^{\prime}\left(x^{-1} g\right), w\right\rangle d \mu(x) \geq 0
$$

because $\mu$ is a positive measure and the integrand is nonnegative. This implies that $\operatorname{ev}(\mu * \chi) \subseteq W^{*}$. Now let $\chi^{\prime}(g) \in$ algint $W^{*}, w \in W \backslash H(W)$ and $U$ be a neighborhood of 1 such that $\left\langle\chi^{\prime}\left(x^{-1} g\right), w\right\rangle>0$ for $x \in U$. Then we have

$$
\left\langle\mu * \chi^{\prime}\left(x_{0} g\right), w\right\rangle \geq \int_{x_{0} U}\left\langle\chi^{\prime}\left(x^{-1} x_{0} g\right), w\right\rangle d \mu(x)>0
$$

because $x^{-1} x_{0} g \in U^{-1} x_{0}^{-1} x_{0} g=U^{-1} g$ implies that the integrand is positive and now $\mu\left(x_{0} U\right)>0$ gives (4).

Lemma II.10. Let $G$ be a connected Lie group and $\chi \in C(G)$. Then we find $f \in C^{\infty}(G)$ such that $f(g)>0$ for all $g \in G, \mu:=f m \in B M^{+}(G)$ ( $m$ is left invariant Haar measure) and $\chi$ is strongly $\mu$-integrable.

Proof. Firstly we choose a compact symmetric neighborhood $K$ of 1 in $G$ and normalize $m$ such that $m(K)=1$. For $j \in \mathbf{N}$ we define

$$
b_{j}:=\max _{x \in K^{j}}|\chi(x)|+1 \quad \text { and } \quad c_{j}:=\frac{1}{2^{j} m\left(K^{j+1}\right) b_{2 j+1}} .
$$

Now we find functions $f_{j} \in C^{\infty}(G)$ with compact support such that $f_{j}(G) \subseteq$ $\left[0, c_{j}\right], f_{j}\left(K^{j}\right)=c_{j}$ and supp $f_{j} \subseteq K^{j+1}$ because int $K^{j+1}$ is an open neighborhood of the compact set $K^{j}$. We set $f:=\sum_{j=1}^{\infty} f_{j}$. For $j \geq i_{0}$ the functions $f_{j}$ are constant on $K^{i_{0}}$ and on this set we have

$$
f=\sum_{j=1}^{i_{0}} f_{j}+\sum_{j=i_{0}+1}^{\infty} f_{j}
$$

where the first summand is in $C^{\infty}(G)$ and the second one is constant on $K^{i_{0}}$. This shows that $f \in C^{\infty}(G)$. From $c_{j}>0$ for all $j \in \mathbf{N}$ and $G=\bigcup_{j=1}^{\infty} K^{j}$ we get the positivity of $f$ on $G$. To prove that $\mu \in B M^{+}(G)$ we compute

$$
\mu(G)=\sum_{j=1}^{\infty} \int_{G} f_{j}(x) d m(x) \leq \sum_{j=1}^{\infty} c_{j} m\left(K^{j+1}\right) \leq \sum_{j=1}^{\infty} \frac{1}{2^{j}}=1 .
$$


Now let $N$ be a compact subset of $G$. Then $N \subseteq K^{i_{0}}$ for some $i_{0} \in \mathbf{N}$ and we find for $i \geq i_{0}$ that

$$
\begin{aligned}
\int_{G} \chi_{N}(x) f_{i}(x) d m(x) & =\int_{K^{i+1}} \chi_{N}(x) f_{i}(x) d m(x) \\
& \leq \int_{K^{i+1}}\left(\max _{y \in K^{i_{0}}} \chi\left(x^{-1} y\right)\right) f_{i}(x) d m(x) \\
& \leq \int_{K^{i+1}}\left(\max _{y \in K^{i_{0}+i+1}} \chi(y)\right) f_{i}(x) d m(x) \\
& \leq b_{i_{0}+i+1} c_{i} m\left(K^{i+1}\right) \leq \frac{b_{i_{0}+i+1}}{b_{2 i+1} 2^{i}} \leq \frac{1}{2^{i}}
\end{aligned}
$$

Summing over $i \in \mathbf{N}$ we get

$$
\int_{G} \chi_{N}(x) d \mu(x) \leq \sum_{i=1}^{i_{0}} \int_{G} \chi_{N}(x) f_{i}(x) d m(x)+\frac{1}{2^{i_{0}}}<\infty
$$

and therefore $\chi$ is strongly $\mu$-integrable because $N$ was arbitrary.

Theorem II.11. Let $G$ be a connected Lie group, $W \subseteq \mathbf{L}(G)$ a Lie wedge, $H \subseteq$ $G$ a closed subgroup with $\mathbf{L}(H)=H(W)$ and $\operatorname{Ad}(\bar{H}) W=W, \pi: G \rightarrow G / \bar{H}$ the canonical projection and $S:=\overline{\langle\exp W\rangle H}$. Then $S$ is a closed submonoid of $G$ and the following assertions are equivalent:

(1) $\mathbf{L}(S)=W$.

(2) For every $\omega \in$ algint $W^{*}$ we find $f \in \operatorname{Mon}(S)$ with $f^{\prime}(\mathbf{1})=\omega$.

(3) There exists $f \in \operatorname{Mon}(S)$ with $f^{\prime}(\mathbf{1}) \in$ algint $W^{*}$.

(4) There exists $f \in \operatorname{Mon}(S)$ with $f^{\prime}(g) \in$ algint $W^{*}$ for all $g \in G$.

(5) There exists $\tilde{f} \in C^{\infty}(G / H)$ with $d \tilde{f}(x) \in \operatorname{int} \theta(x)^{*}$ for all $x \in M$, where $\theta(\pi(g))=d \pi(g) d \lambda_{g}(\mathbf{1}) W$ for all $g \in G$.

Proof. That $S$ is a submonoid of $G$ follows from

$$
h\langle\exp W\rangle h^{-1}=\langle\exp \operatorname{Ad}(h) W\rangle=\langle\exp W\rangle \text { for all } h \in H .
$$

$(1) \Rightarrow(2)$. This is Lemma I.8.

$(2) \Rightarrow(3)$. This is trivial.

$(3) \Rightarrow(4)$. Let $f_{1} \in \operatorname{Mon}(S)$ with $f^{\prime}(\mathbf{1}) \in$ algint $W^{*}$ and

$$
\chi(g):=\max \left(\left|f_{1}(g)\right|,\left\|f_{1}^{\prime}(g)\right\|\right) \text {. }
$$

Then $\chi \in C(G)$ and with Lemma II.10 we find $f_{0} \in C^{\infty}(G)$ such that $f_{0}(g)>$ 0 for all $g \in G$, the measure $\mu=f_{0} m$ is in $B M^{+}(G)$, where $m$ is left invariant Haar measure, and $\chi$ is strongly $\mu$-integrable. Therefore $f_{1}$ and $f_{1}^{\prime}$ are strongly $\mu$-integrable. From Proposition II.9 we get $f_{2}:=\mu * f_{1} \in C^{1}(G, \mathbf{R})$ and $f_{2}^{\prime}=\mu * f_{1}^{\prime}$. Applying II.9(3) we see that

$$
f_{2}^{\prime}\left(x_{0}\right) \in \text { algint } W^{*} \text { for all } x_{0} \in G
$$

because $f_{1}^{\prime}(1) \in$ algint $W^{*}$ and $\operatorname{supp} \mu=G$, i.e. every open subset of $G$ has positive $\mu$-measure. Finally we find $f_{3} \in C^{\infty}(G)$ with compact support such that $f_{3} \geq 0$ and $f_{3}(\mathbf{1})>0$. Then $f=f_{3} * f_{2} \in C^{\infty}(G)$ is the desired function. To see this, we remark that $f^{\prime}\left(x_{0}\right) \in$ algint $W^{*}$ for all $x_{0} \in G$ because $1 \in$ $\operatorname{supp}\left(f_{3} m\right)$ (Proposition II.9(3)). 
(4) $\Leftrightarrow(5)$. Every function $f \in \operatorname{Mon}(S)$ is constant on the cosets $g H$ of $H$ and therefore we find $\tilde{f} \in C^{\infty}(G / H)$ with $f=\tilde{f} \circ \pi$. The rest follows from Lemma II.5.

$(4) \Rightarrow(1)$. Let $f \in \operatorname{Mon}(S)$ such that $f^{\prime}(g) \in$ algint $W^{*}$ for all $g \in G$ and $V=\operatorname{ev}(\operatorname{Pos}(W))$. Using Proposition I.6 we have to show that $V^{*}=W$. To every functional $\omega \in$ algint $W^{*}$ we find a function $\tilde{f}_{1}$ on a neighborhood $\pi(U)$ of $\pi(\mathbf{1}) \in G / H$ such that $\tilde{f}_{1} \in \operatorname{Pos}_{G / H, U}(W)$ and $d \tilde{f}_{1}(\pi(\mathbf{1})) d \pi(\mathbf{1})=\omega$. Now Corollary II.6 gives us a function $f_{2} \in \operatorname{Pos}(W)$ such that $f_{2}^{\prime}(\mathbf{1})=d\left(\tilde{f}_{1} \circ \pi\right)(\mathbf{1})=$ $\omega$. This shows that algint $W^{*} \subseteq V$ and therefore

$$
W=\left(\text { algint } W^{*}\right)^{*} \supseteq V^{*} \supseteq W^{* *}=W .
$$

Theorem II.12 (Characterization of globality). Let $G$ be a connected Lie group $W \subseteq \mathbf{L}(G)$ a Lie wedge and $H=\langle\exp H(W)\rangle$. Then the following assertions are equivalent:

(1) $\mathbf{L}(\overline{\langle\exp W\rangle})=W$.

(2) $H$ is closed and for every $\omega \in$ algint $W^{*}$ we find $f \in \operatorname{Pos}(W)$ such that $f^{\prime}(\mathbf{1})=\omega$.

(3) $H$ is closed and we find $f \in \operatorname{Pos}(W)$ such that $f^{\prime}(\mathbf{1}) \in$ algint $W^{*}$.

(4) $H$ is closed and we find $f \in \operatorname{Pos}(W)$ such that $f^{\prime}(g) \in$ algint $W^{*}$ for all $g \in G$.

Suppose that $W$ is Lie generating in $\mathbf{L}(G)$, then these conditions are equivalent to the globality of $W$ in $G$.

Proof. Firstly we notice that $H \subseteq\langle\exp W\rangle$. Consequently $S:=\overline{\langle\exp W\rangle H}=$ $\overline{\langle\exp W\rangle}$.

(1) $\Rightarrow$ (2). We firstly show that $H$ is closed. We have $\mathbf{L}(S)=W$. Therefore $\bar{H} \subseteq S$ and hence $\mathbf{L}(\bar{H}) \subseteq \mathbf{L}(S)=W$. But $H(W)$ is the largest vector space contained in $W$, therefore $H=\bar{H}$ because $\mathrm{L}(H)=H(W)=\mathbf{L}(\bar{H})$. The rest follows immediately from Lemma I.8.

(2) $\Rightarrow(3) \Rightarrow(4) \Rightarrow(1)$. These are consequences of Theorem II.11 because $H$ is assumed to be closed and

$$
\operatorname{Pos}(W)=\operatorname{Mon}(S)=\operatorname{Mon}(\langle\exp W\rangle) .
$$

The last statement is clear from the definition of the tangent wedge of a subsemigroup $S$ of $G$ (Introduction).

Corollary II.13. Let $W$ be a Lie generating Lie wedge in the Lie algebra of the connected Lie group $G$. Then the following are equivalent

(1) $\langle\exp W\rangle \neq G$.

(2) $\operatorname{Pos}(W)$ contains a nonconstant function.

Proof. (1) $\Rightarrow(2)$ : Let $S=\langle\exp W\rangle$. We know from [7, V.1.16] that int $S \neq$ $\varnothing$. We claim that $S \cap$ int $S^{-1}=\varnothing$ which implies that int $S^{-1} \cap \bar{S}=\varnothing$ and $\bar{S} \neq G$. For $x \in \operatorname{int} S^{-1} \cap S$ we have $\mathbf{1}=x x^{-1} \in S$ int $S \subseteq$ int $S$ and therefore $G=S$ because $G$ is connected, a contradiction which proves our claim. But from $\bar{S} \neq G$ we get $\mathbf{L}(S) \neq \mathbf{L}(G)$ and Theorem II.12 provides a function $f \in \operatorname{Pos}(\mathbf{L}(S)) \subseteq \operatorname{Pos}(W)$ with $f^{\prime}(\mathbf{1}) \neq 0$. Then $f$ is the desired function.

$(2) \Rightarrow(1)$. Let $f \in \operatorname{Pos}(W)$ be a nonconstant function. Because $G$ is connected we find $g \in G$ with $f(g) \neq 0$. Then $g$ is a regular point of $f$ and 
$f(G)$ is a neighborhood of $f(g)$ in $\mathbf{R}$. But we clearly have that

$$
g S=g(\exp W\rangle \subseteq\{x \in G: f(x) \geq f(g)\} \neq G
$$

because $f(g S) \neq f(G)$.

\section{Consequences of the Characterization Theorem}

Proposition III.1 [7, VI.5.2]. Let $G$ be a connected Lie group and $W_{1} \subseteq W_{2}$ two Lie generating Lie wedges in $\mathbf{L}(G)$. Then the following conditions are sufficient for $W_{1}$ to be global in $G$ :

(1) $W_{2}$ is global in $G$,

(2) $W_{1} \cap H\left(W_{2}\right) \subseteq H\left(W_{1}\right)$, and

(3) the analytic subgroup generated by $\exp H\left(W_{1}\right)$ is closed in $G$.

Proof. From $W_{1} \subseteq W_{2}$ we get $\operatorname{Pos}\left(W_{2}\right) \subseteq \operatorname{Pos}\left(W_{1}\right)$. According to Theorem II.12 we find $f \in \operatorname{Pos}\left(W_{2}\right)$ such that $f^{\prime}(\mathbf{1}) \in$ algint $W_{2}^{*}$. But for $w \in$ $W_{1} \backslash H\left(W_{1}\right) \subseteq W_{2} \backslash H\left(W_{2}\right)$ we have $\left\langle f^{\prime}(\mathbf{1}), w\right\rangle>0$ and therefore $f^{\prime}(\mathbf{1}) \in$ algint $W_{1}$. Now an application of Theorem II.12 completes the proof.

Remark III.2. If the Lie wedge $W_{1}$ is global in $G$, Lie generating, and $f \in$ $\operatorname{Pos}\left(W_{1}\right)$ such that $f^{\prime}(1) \in$ algint $W_{1}^{*}$, then $W_{2}=\operatorname{ev}(f)^{*}$ is a global Lie wedge which contains $W_{1}$ and which satisfies

$$
W_{1} \cap H\left(W_{2}\right)=H\left(W_{1}\right) .
$$

This follows easily from the fact that $w \in W_{1} \cap H\left(W_{2}\right)$ implies $\left\langle f^{\prime}(g), w\right\rangle=0$ for all $g \in G$, especially for 1 , which shows that $w \in H\left(W_{1}\right)$

Proposition III.3. Let $G$ be a Lie group, $W \subseteq \mathbf{L}(G), f \in \operatorname{Pos}(W)$ and $K \subseteq G$ be a compact subgroup. Let $\mu \in B M^{+}(G)$ be defined by $\mu(M)=m(M \cap K)$ where $m$ is normalized Haar measure on $K$. Then $\mu * f \in \operatorname{Pos}(W)$ and this function is constant on the cosets $K g$ for $g \in G$. If $f^{\prime}(g) \in$ algint $W^{*}$, then

$$
\mu * f^{\prime}(g) \in \text { algint } W^{*} .
$$

Proof. We apply Proposition II.9. According to the remark in Definition II.8 all functions $f^{(n)}$ for $n \in \mathbf{N}$ are strongly $\mu$-integrable. Therefore $\mu * f \in C^{\infty}(G)$. The rest follow from Proposition II.9(3) because $1 \in \operatorname{supp}(\mu)$.

Proposition III.4. Let $G$ be a connected Lie group, $K \subseteq \operatorname{Aut}(G)$ be a compact subgroup, $m$ normalized Haar measure on $K, W \subseteq \mathbf{L}(G)$ a Lie wedge which is invariant under $d k(\mathbf{1})$ for every $k \in K$, and $f \in \operatorname{Pos}(W)$. Then the function $\tilde{f}$ defined by

$$
\tilde{f}(g)=\int_{K} f(k \cdot g) d m(k)
$$

is in $\operatorname{Pos}(W)$ and satisfies $\tilde{f} \circ k=\tilde{f}$ for all $k \in K$. If $f^{\prime}(g) \in$ algint $W^{*}$, then

$$
\tilde{f}^{\prime}(g) \in \text { algint } W^{*} \text {. }
$$

Proof. By $[1$, p. 251] the mapping $K \times G \rightarrow G,(k, g) \mapsto k(g)=k . g$ is analytic and because $K$ is compact we may differentiate under the integral to get

$$
\begin{aligned}
\tilde{f}^{\prime}(g) & =\int_{K} d(f \circ k)(g) d \lambda_{g}(\mathbf{1}) d m(k)=\int_{K} d f(k . g) d k(g) d \lambda_{g}(\mathbf{1}) d m(k) \\
& =\int_{K} d f(k . g) d \lambda_{k . g}(\mathbf{1}) d k(\mathbf{1}) d m(k)=\int_{K} f^{\prime}(k \cdot g) \circ d k(\mathbf{1}) d m(k) .
\end{aligned}
$$


Now we see that $\tilde{f} \in \operatorname{Pos}(W)$ because $d k(\mathbf{1}) W=W$ for every $k \in K$. Let $f^{\prime}(g) \in$ algint $W$ and $w \in W \backslash H(W)$. Then we have

$$
\left\langle f^{\prime}(g), w\right\rangle=\int_{K}\left\langle f^{\prime}(k . g) d k(\mathbf{1}), w\right\rangle d m(k)>0
$$

because the integrand is nonnegative and positive at $k=1$.

Proposition III.5. Let $G$ be a connected Lie group, $W \subseteq \mathbf{L}(G)$ a Lie generating Lie wedge and $K \subseteq G$ a compact subgroup. Then

$$
\mathbf{L}(K) \cap W \subseteq H(W)
$$

if $W$ is global in $G$. Suppose, in addition to (5), that $\operatorname{Ad}(K) W=W$. Then the Lie wedge $V:=W+\mathbf{L}(K)$ is global in $G$ iff $W$ is global in $G$.

Proof. Let $w \in W \cap \mathbf{L}(K)$ and assume that $W$ is global in $G$. According to Theorem II.12 we find $f \in C^{\infty}(G)$ such that $f^{\prime}(g) \in$ algint $W^{*}$ for all $g \in G$, especially for $g \in K$. Now $K$ is compact and the function $\left.f\right|_{K}$ has a maximum on $g_{0} \in K$. Therefore $\left\langle f^{\prime}\left(g_{0}\right), \mathbf{L}(K)\right\rangle=\{0\}$ which shows that $w \in H(W)$. Suppose that $W$ is invariant under $\operatorname{Ad}(K)$. From (5) and [7, I.2.32] we get that $V$ is a wedge. For $h \in H(V)=H(W)+\mathbf{L}(K)$ we clearly have that $e^{\text {ad } h} V=V$ and therefore $V$ is a Lie wedge. Using Propositions III.3 and 4 we find a function $f \in \operatorname{Pos}(W)$ such that $f^{\prime}(\mathbf{1}) \in$ algint $W^{*}, f \circ I_{k}=f$ for all $k \in K$ and $f(K g)=\{f(g)\}$ for all $g \in G$. Therefore $f(g K)=\{f(g)\}$ for all $g \in G$. This leads to $f^{\prime}(g) \in \mathbf{L}(K)^{\perp}$ for all $g \in G$ and consequently $f \in \operatorname{Pos}(V)$ and $f^{\prime}(\mathbf{1}) \in$ algint $W^{*} \cap \mathbf{L}(K)^{\perp}=$ algint $V^{*}$. An application of Theorem II.12 finishes the proof. The other direction can directly be obtained by Lemma III. 1 .

Proposition III.6. Let $G$ be a connected Lie group, $H \subseteq G$ a closed connected subgroup of codimension 1 , and $W \subseteq \mathbf{L}(G)$ a Lie wedge with $H(W)=\mathbf{L}(H)$. Then $W$ is global in $G$ iff $G / H$ is not compact, i.e. diffeomorphic to the circle. The conditions are satisfied if $G$ is simply connected.

Proof. If $G$ is simply connected and $H$ is connected, then $G / H$ is also simply connected and therefore diffeomorphic to $\mathbf{R}$. Assume this. Let $\pi: G \rightarrow G / H$ be the canonical projection and $\tilde{f}: G / H \rightarrow \mathbf{R}$ be a diffeomorphism such that $d \tilde{f}(d \pi(\mathbf{1}) W) \subseteq \mathbf{R}^{+}$. For $f:=\tilde{f} \circ \pi$ we conclude that $f^{\prime}(g) \in H(W)^{\perp} \backslash\{0\}$ which is the union of the two open half lines algint $W^{*}$ and - algint $W^{*}$. Because $f$ has no singular points and $f^{\prime}(\mathbf{1}) \in W^{*}$ we get $f^{\prime}(g) \in$ algint $W^{*}$ for all $g \in G$ because $G$ is connected. Theorem II.12 is now applicable and proves that $W$ is global. If $G / H$ is compact it is impossible to find a function $f \in C^{\infty}(G / H)$ without singular points, hence $W$ is not global in $G$ (Theorem II.11).

Lemma III.7. Let $p: \widetilde{G} \rightarrow G$ be a morphism of connected Lie groups and $W \subseteq$ $\mathbf{L}(G)$ be Lie generating and global in $G$. Then $V:=d p(\mathbf{1})^{-1}(W)$ is global in $\widetilde{G}$. 
Proof. Let $S \subseteq G$ be a closed subsemigroup of $G$ such that $\mathbf{L}(S)=W$ and $\widetilde{S}:=p^{-1}(S)$. Then $\widetilde{S} \subseteq \widetilde{G}$ is a closed subsemigroup and

$$
\begin{aligned}
\mathbf{L}(\widetilde{S}) & =\left\{x \in \mathbf{L}(\widetilde{G}): \exp \left(\mathbf{R}^{+} x\right) \subseteq \widetilde{S}\right\} \\
& =\left\{x \in \mathbf{L}(\widetilde{G}): p\left(\exp \left(\mathbf{R}^{+} x\right)\right) \subseteq S\right\} \\
& =\left\{x \in \mathbf{L}(\widetilde{G}): \exp \left(\mathbf{R}^{+} d p(\mathbf{1}) x\right) \subseteq S\right\} \\
& =d p(\mathbf{1})^{-1}\left\{x \in \mathbf{L}(G): \exp \left(\mathbf{R}^{+} x\right) \subseteq S\right\}=d p(\mathbf{1})^{-1} \mathbf{L}(S)=V .
\end{aligned}
$$

Hence $V$ is global in $\widetilde{G}$.

Lemma III.8. Let $G$ be a connected Lie group, $W \subseteq \mathbf{L}(G)$ be a Lie generating Lie wedge, $S:=\overline{\langle\exp W\rangle}$, and $H \subseteq H(S)$ a closed normal subgroup of $G$. Then $W / \mathbf{L}(H)$ is global in $G / H$ iff $W$ is global in $G$.

Proof. That the globality of $W / \mathbf{L}(H)$ implies the globality of $W$ follows from Lemma III.7. Let $p: G \rightarrow G / H$ be the canonical projection and $T:=p(S)$. The closedness of $T$ follows from $S H=S$ and the closedness of $S \subseteq G$. Let $W=\mathbf{L}(S)$ be global in $G$. Then we find that

$$
\begin{aligned}
\mathbf{L}(T) & =\left\{x \in \mathbf{L}(\widetilde{G}): \exp \left(\mathbf{R}^{+} x\right) \subseteq T\right\} \\
& =\left\{x \in \mathbf{L}(\widetilde{G}): p^{-1}\left(\exp \left(\mathbf{R}^{+} x\right)\right) \subseteq S\right\} \\
& =d p(\mathbf{1})\left\{y \in \mathbf{L}(G): \exp \left(\mathbf{R}^{+} y\right) \subseteq S\right\} \\
& =d p(\mathbf{1}) \mathbf{L}(S)=W / \mathbf{L}(H) .
\end{aligned}
$$

Therefore $W / \mathbf{L}(H)$ is global in $\widetilde{G}$.

Proposition III.9. Let $G$ be a connected Lie group and $W \subseteq \mathbf{L}(G)$ a Lie wedge which is invariant under the adjoint action, $H:=\langle\exp H(\bar{W})\rangle$ the normal analytic subgroup of $G$ generated by the ideal $H(W)$ and $p: G \rightarrow G / H$ the canonical projection. Suppose that $H$ is closed in $G$. Then $W$ is global in $G$ if and only if the pointed invariant Lie wedge $V:=d p(1) W$ is global in $G / H$.

Proof. This is a consequence of Lemma III.8.

Proposition III.10. Let $G$ be a connected Lie group, $W \subseteq \mathbf{L}(G)$ be a Lie generating Lie wedge and $N \subseteq G$ a closed connected subgroup containing the commutator group $G^{\prime}=(G, G)$. Then the following conditions are sufficient for $W$ to be global in $G$ :

(1) $H:=\langle\exp W\rangle$ is closed in $G$,

(2) the fundamental group $\pi_{1}(G / N)$ is finite, and

(3) $W \cap \mathbf{L}(N) \subseteq H(W)$.

Proof. From [7, I.2.32] we know that $V:=W+\mathbf{L}(N)$ is a wedge. But $H(V)$ contains the commutator algebra $L^{\prime}=[L, L]$, and therefore it has to be a Lie wedge because for $v \in V$ and $x \in L$ we have $e^{\text {ad } x} v \subseteq v+L^{\prime} \subseteq V$. The factor group $G / N$ is an abelian Lie group with finite fundamental group, hence a vector group. Consequently the wedge $V / \mathbf{L}(N)$ is global in $G / N$. According to Proposition III.9 the wedge $V$ is global in $G$. Now Proposition III.1 implies the globality of $W$ in $G$ because $W \cap H(V) \subseteq H(W)$ and $H$ is closed. 
Proposition III.11. Let $G$ be a connected Lie group with compact Lie algebra $L=\mathbf{L}(G)$ and $W$ a Lie generating Lie wedge in $L$. Suppose that $H:=$ $\langle\exp H(W)\rangle$ is closed in $G$. Then $W$ is global in $G$ iff

$$
\mathbf{L}(K) \cap W \subseteq H(W)
$$

for every compact subgroup $K \subseteq G$.

Proof. The necessity follows from Proposition III.5. To prove sufficiency we notice that the Lie algebra $L$ is reductive and therefore $L=Z(L) \oplus[L, L]$ where $L^{\prime}=[L, L]$ is a compact semisimple Lie algebra. According to $[8, \mathrm{p}$. 180] we find an $n \in \mathbf{N}$ and a maximal compact subgroup $K \subseteq G$ such that $G$ is diffeomorphic to $K \times \mathbf{R}^{n}$. Consequently $K$ is connected. The subgroup $G^{\prime}=\left\langle\exp L^{\prime}\right\rangle$ of $G$ is compact, semisimple and normal. This implies that $G^{\prime} \subseteq K$ because every compact subgroup is conjugate to a subgroup of $K$ under an inner automorphism of $G$. The manifold $G / K$ is diffeomorphic to $n$-space and has finite fundamental group. An application of Proposition III.10 completes the proof.

In the following we shall refer to the Campbell-Hausdorff multiplication in a Lie algebra: $x * y=x+y+\frac{1}{2}[x, y] \ldots$.

Proposition III.12. Let $G$ be a connected Lie group with exponential Lie algebra $L$ and $W \subseteq L$ a Lie semialgebra, i.e. a wedge satisfying $W * W \subseteq W$. Then $W$ is global in $G$ iff $H:=\langle\exp H(W)\rangle$ is closed in $G$ and

$$
\mathbf{L}(K) \cap W \subseteq H(W)
$$

for every compact subgroup $K \subseteq G$.

Proof. According to our assumption that $L$ is exponential, we find a global continuation of the Campbell-Hausdorff multiplication to $L \times L$. We write $*$ for this continuation, too. Then $(L, *)$ is a simply connected Lie group and the exponential function exp: $L \rightarrow G$ is a covering morphism of Lie groups. The subgroup $D=\operatorname{ker}(\exp )$ is central and discrete in $(L, *)$. We claim that $D \subseteq Z(L)$, the center of the Lie algebra $L$. Using $e^{\text {ad } d}=\mathrm{id}_{L}$ we find that ad $d$ is semisimple and $\operatorname{Spec}(\operatorname{ad} d) \subseteq 2 \pi i Z$. But the exponential function $\exp _{L}$ is a diffeomorphism and has no singular points which implies that $\operatorname{Spec}(\operatorname{ad} d)=\{0\}$ and ad $d=0[6$, p. 105]. This proves our claim. Now we have for $x \in L$ and $d \in D$ that $x * d=x+d$. Then $A:=\operatorname{span} D \subseteq Z(L)$ and $\exp _{G} A$ is a compact subgroup of $G$. Our assumption leads to $A \cap W \subseteq H(W)$. Using Propositions I.2.32, II.2.42 and II.4.1 in [7] we find that $V:=W+A$ is a semialgebra in $L$, i.e. a subsemigroup of $(L, *)$ with $\mathbf{L}(V)=V$, hence global in $(L, *)$. The globality of $V$ in $G$ follows from Lemma III.8 and $D \subseteq A \subseteq H(V)$. To complete the proof we notice that $W \cap H(V)=W \cap(H(W)+A) \subseteq H(W)$ and apply Proposition III.1.

Remark III.13. The abelian and nilpotent Lie algebras are special classes of exponential Lie algebras. It is also easy to see that every exponential Lie algebra is solvable. 


\section{IV. $W$-POSITIVE FUNCTIONS}

At this stage we describe the relations between $f$ and $\operatorname{ev}(f)$ in greater detail.

Proposition IV.1. Let $f \in C^{\infty}(G)$ be a nonconstant function, i.e. $\operatorname{ev}(f) \neq\{0\}$, with $f(\mathbf{1})=0$. Then the following assertions hold.

(1) If $\alpha \in \operatorname{Aut}(G)$ and $f=f \circ \alpha$, then

$$
\widehat{d \alpha(\mathbf{1})} \operatorname{ev}(f) \subseteq \operatorname{ev}(f) \text { and } d \alpha(\mathbf{1}) \operatorname{ev}(f)^{*} \subseteq \operatorname{ev}(f)^{*} .
$$

(2) $\operatorname{ev}(f)^{*}=\mathbf{L}(\mathbf{S G}(f))$ is a global Lie wedge.

(3) For an analytic subgroup $H \subseteq G$ the function $f$ is constant on the cosets $g H$ for all $g \in G$ iff $\operatorname{ev}(f) \subseteq \mathbf{L}(H)^{\perp}$.

(4) $\operatorname{ev}(f)=\{\omega\}$ iff $f: G \rightarrow \mathbf{R}$ is a morphism of Lie groups. In this case $\operatorname{ev}(f)^{*}=\omega^{-1}\left(\mathbf{R}^{+}\right)$is a half space whose boundary is an ideal in $\mathbf{L}(G)$ and $\omega=d f(\mathbf{1}): \mathbf{L}(G) \rightarrow \mathbf{R}$ is a morphism of Lie algebras.

(5) $\operatorname{dim} \operatorname{ev}(f) \leq 1$ iff $\operatorname{ev}(f)^{*}$ is a global half space semialgebra, i.e. a half space whose boundary is a Lie algebra.

Proof. (1) We only have to show that $\widehat{d \alpha(\mathbf{1})} \operatorname{ev}(f) \subseteq \operatorname{ev}(f)$. For $g, h \in G$ we have that

$$
\begin{aligned}
& \widehat{d \alpha(\mathbf{1})} f^{\prime}(g)=d f(g) d \lambda_{g}(\mathbf{1}) d \alpha(\mathbf{1})=d f(g) d \alpha\left(\alpha^{-1}(g)\right) d \lambda_{\alpha^{-1}(g)}(\mathbf{1}) \\
& =d(f \circ \alpha)\left(\alpha^{-1}(g)\right) d \lambda_{\alpha^{-1}(g)}(\mathbf{1})=d f\left(\alpha^{-1}(g)\right) d \lambda_{\alpha^{-1}(g)}(\mathbf{1}) \\
& =f^{\prime}\left(\alpha^{-1}(g)\right) \in \operatorname{ev}(f) .
\end{aligned}
$$

(2) This is a special case of Lemma I.3.

(3) $f$ is constant on all cosets $g H$ iff $f$ is constant along all curve $t \mapsto$ $g \exp (t h)$ for $h \in \mathbf{L}(H)$. But this is clearly equivalent to

$$
d f(g) d \lambda_{g}(\mathbf{1}) \mathbf{L}(H)=f^{\prime}(g) \mathbf{L}(H)=\{0\} \text { for all } g \in G .
$$

(4) Let $\operatorname{ev}(f)=\{\omega\}$ and $g \in G$. Then $\omega \neq 0$ because $f$ is not constant and $d f$ is a left-invariant closed 1 -form on $G$. This leads to

$$
d\left(f \circ \lambda_{g}\right)(x)=d f(g x) \circ d \lambda_{g}(x)=d f(x)=d(f+f(g))(x)
$$

and $f \circ \lambda_{g}(\mathbf{1})=f(g)=f(\mathbf{1})+f(g)$ which proves that $f \circ \lambda_{g}=f+f(g)$ because $G$ is connected. Applying this to the argument $h \in G$ we find that $f(g h)=f(g)+f(h)$, i.e. $f$ is a morphism of Lie groups. If $f: G \rightarrow \mathbf{R}^{+}$is homomorphic, we get $f \circ \lambda_{g}=f+f(g)$ for all $g \in G$ and differentiation yields

$$
f^{\prime}(g)=d f(g) d \lambda_{g}(\mathbf{1})=d f(\mathbf{1})=\omega \text { for all } g \in G .
$$

(5) The zero dimensional case is described in (3). If $\operatorname{ev}(f)$ is one dimensional, we deduce from $(2)$ that $\operatorname{ev}(f)^{*}$ is a global half space Lie wedge whose boundary has to be a subalgebra of $\mathbf{L}(G)$. If $\operatorname{ev}(f)^{*}$ is a half space semialgebra, then $\operatorname{ev}(f) \subseteq \operatorname{ev}(f)^{* *}=\mathbf{R}^{+} f^{\prime}(g)$ if $f^{\prime}(g) \neq 0$. Consequently $\operatorname{ev}(f)$ is at most one dimensional.

An interesting source of functions which determine global Lie wedges is described in the following proposition. 
Proposition IV.2. Let $G$ be a connected Lie group, $V$ a Fréchet space, $\rho$ a continuous representation of $G$ on $V, \omega \in V^{*}, V_{+}:=\{\hat{\rho}(G) \omega: g \in G\}^{*}$ and $\Phi_{\omega, x}(g):=\langle\omega, \rho(g) x\rangle$. Suppose that $\Phi_{\omega, x} \in C^{\infty}(G)$, then

$$
\mathbf{L}\left(\mathbf{S G}\left(\Phi_{\omega, x}\right)\right)=\left\{y \in \mathbf{L}(G): d \rho(\mathbf{1})(y) x \in V_{+}\right\} .
$$

Proof. It is clear that $V_{+}$is a $G$-invariant wedge in $V$ and $\operatorname{SG}\left(\Phi_{\omega, x}\right)=$ $\left\{s \in G: \rho(s) x-x \in V_{+}\right\}$because $\boldsymbol{\Phi}_{\omega, x}(g s) \geq \Phi_{\omega, x}(g)$ is equivalent to $\langle\hat{\rho}(g) \omega, \rho(s) x\rangle \geq\langle\hat{\rho}(g) \omega, x\rangle$. Using Lemma I.3 we get $\mathbf{L}\left(\operatorname{SG}\left(\Phi_{\omega, x}\right)\right)=$ $\operatorname{ev}\left(\Phi_{\omega, x}^{\prime}\right)^{*}$ and for $g \in G$ we have

$$
\left\langle\Phi_{\omega, x}^{\prime}(g), y\right\rangle=\langle\hat{\rho}(g) \omega, d \rho(\mathbf{1})(y) x\rangle .
$$

Combining these two assertions leads to

$$
\mathbf{L}\left(\mathbf{S G}\left(\boldsymbol{\Phi}_{\omega, x}\right)\right)=\operatorname{ev}\left(\boldsymbol{\Phi}_{\omega, x}\right)^{*}=\left\{y \in \mathbf{L}(G): d \rho(\mathbf{1})(y) x \in V_{+}\right\} .
$$

Remark IV.3. If the wedge $V_{+}$is not pointed one may factor the edge $H\left(V_{+}\right)$ and get a representation of $G$ in the factor space $V_{1}=V / H\left(V_{+}\right)$. If $\tilde{\omega} \in \widehat{V}_{1}$ is such that $\omega=\tilde{\omega} \circ \pi$ where $\pi: V \rightarrow V_{1}$ is the projection, then we have

$$
\operatorname{SG}\left(\Phi_{\omega, x}\right)=\operatorname{SG}\left(\Phi_{\tilde{\omega}, \pi(x)}\right)
$$

which follows from the fact that $\langle\omega, \rho(s) x-x\rangle \geq 0$ is equivalent to

$$
\langle\tilde{\omega}, \pi(\rho(s) x)-\pi(x)\rangle \geq 0 .
$$

Example IV.4. Let $V=C^{\infty}(G)$ with its Fréchet topology, $\rho$ the right-regular representation of $G, \omega=\delta_{1} \in \widehat{V}$ and $f \in V$. Then

$$
\boldsymbol{\Phi}_{\omega, f}(g)=\left\langle\delta_{1}, \rho(g) f\right\rangle=f(g) \text { and } d \rho(\mathbf{1})(y) f=\left\langle f^{\prime}(\mathbf{1}), y\right\rangle \text {. }
$$

Example IV.5. Let $V=\operatorname{sl}(2, \mathbf{R}), G=\mathrm{Sl}(2, \mathbf{R})$ and $\rho=\mathrm{Ad}$. We set $\mathbf{R}^{+}=$ $[0, \infty[$ and use the same notations as in [7] for the elements of $V$

$$
H=\left(\begin{array}{cc}
1 & 0 \\
0 & -1
\end{array}\right), \quad T=\left(\begin{array}{ll}
0 & 1 \\
1 & 0
\end{array}\right), \quad \text { and } \quad U=\left(\begin{array}{cc}
0 & 1 \\
-1 & 0
\end{array}\right) \text {. }
$$

These matrices satisfy the relations

$$
[U, T]=2 H, \quad[U, H]=-2 T, \quad \text { and } \quad[H, T]=2 U .
$$

We identify $\widehat{V}$ and $V$ using the Cartan Killing form $B(h H+t T+x U)=$ $8\left(h^{2}+t^{2}-x^{2}\right)$. For $\omega=U$ we get $V_{+}=\left\{x U+h H+t T: x \geq 0, h^{2}+t^{2} \leq x^{2}\right\}$ and for $A \in V$, therefore,

$$
\mathbf{L}\left(\operatorname{SG}\left(\Phi_{U, A}\right)\right)=\left\{Y \in \operatorname{sl}(2, \mathbf{R}):[Y, A] \in V_{+}\right\} .
$$

There are three essentially different cases.

(1) $A=U: \mathbf{L}\left(\mathbf{S G}\left(\Phi_{U, A}\right)\right)=\mathbf{R} U$,

(2) $A=U+H: \mathbf{L}\left(\mathbf{S G}\left(\Phi_{U, A}\right)\right)=\mathbf{R}(U+H)-\mathbf{R}^{+} T$,

(3) $A=H: \mathbf{L}\left(\mathbf{S G}\left(\Phi_{U, A}\right)\right)=\mathbf{R} H+\mathbf{R}^{+}(U-T)-\mathbf{R}^{+}(U+T)$.

Using the above relations for the brackets of $H, T$ and $U$, the verifications of (1)-(3) are simple computations.

We conclude this paper with a description of a useful method to construct $W$-positive functions. We call it the method of adapted functions. We first need some information about left-invariant 1 -forms on a Lie group $G$. 
Lemma IV.6. Let $G$ be a connected Lie group, $L=\mathbf{L}(G)$ its Lie algebra and $\omega$ a nonzero left-invariant 1-form on $G$. Then the following assertions are true:

(1) $\omega$ is closed iff $\omega(\mathbf{1}) \in[L, L]^{\perp}$.

(2) Suppose that $\omega$ is closed. Then $\omega$ is exact iff the normal analytic subgroup $A \subseteq G$ with $\mathbf{L}(A)=\operatorname{ker} \omega(\mathbf{1})$ is closed in $G$ and $G / A \cong \mathbf{R}$. These conditions are fulfilled if $G$ is simply connected.

Proof. (1) Let $x, y \in \mathbf{L}(G)$ and $X, Y$ be two left-invariant vector fields on $G$ with $X(\mathbf{1})=x$ and $Y(\mathbf{1})=y$. Then we have for $g \in G$ that

$$
\begin{aligned}
d \omega(g)(X, Y) & =X\langle\omega, Y\rangle(g)-Y\langle\omega, X\rangle(g)-\langle\omega(g),[X, Y](g)\rangle \\
& =X\langle\omega(\mathbf{1}), y\rangle(g)-Y\langle\omega(\mathbf{1}), x\rangle(g)-\langle\omega(\mathbf{1}),[x, y]\rangle \\
& =\langle\omega(\mathbf{1}),[y, x]\rangle .
\end{aligned}
$$

Now it is immediate that the closedness of $\omega$ is equivalent to the vanishing of $\omega(\mathbf{1})$ on the commutator algebra.

(2) Suppose that $\omega$ is exact and $d f=\omega$ with $f(\mathbf{1})=0$. From Proposition IV.1 we see that $f: G \rightarrow \mathbf{R}$ is a surjective morphism of Lie groups. Therefore $A=(\operatorname{ker} f)_{0}$ is closed and connected in $G$, and $G / A \cong f(G)=\mathbf{R}$. To prove the other direction we assume that $A$ is closed and $G / A \cong \mathbf{R}$. Let $f: G \rightarrow G / A$ be the quotient morphism. Then $f$ is a morphism of Lie groups because $A$ is normal in $G$ and $d f(\mathbf{1})=\lambda \omega(\mathbf{1})$ for some $\lambda \neq 0$. But $d f$ and $\omega$ are leftinvariant 1-forms (Proposition IV.1) which shows that $d f=\lambda \omega$ and therefore $\omega=d(f / \lambda)$ is exact. It is a standard fact from Lie theory that if $G$ is simply connected, then every normal analytic subgroup of $G$ has to be closed and the quotients are also simply connected.

Definition IV.7. Let $G$ be a connected Lie group and $A$ and $B$ closed analytic subgroups of $G$. We say that $G=A B$ is a product decomposition if the mapping $m: A \times B \rightarrow G,(a, b) \mapsto a b$ is a diffeomorphism. We call a function $f \in$ $C^{\infty}(G)$ adapted (to that decomposition) if we have

$$
f(a g b)=f(a)+f(g)+f(b) \text { for all } a \in A, b \in B \text { and } g \in G .
$$

Example IV.8. Let $G$ be a connected semisimple Lie group and $\mathbf{L}(G)=K \oplus T$ an Iwasawa decomposition, where $T$ is solvable and $K$ is maximal compactly embedded. Then $G=\langle\exp T\rangle\langle\exp K\rangle$ is a product decomposition [6, p. 270].

Lemma IV.9. Let $G=A B$ be a product decomposition. Then the function $f \in$ $C^{\infty}(G)$ is adapted to the decomposition iff $f \circ m: A \times B \rightarrow \mathbf{R}$ is a homomorphism of Lie groups, where $A \times B$ is the direct product of the Lie groups $A$ and $B$.

Proof. Firstly we assume that $f \circ m: A \times B \rightarrow \mathbf{R}$ is a homomorphism. For $g=a^{\prime} b^{\prime}, a \in A$ and $b \in B$ we have

$$
\begin{aligned}
f(a g b) & =f\left(a a^{\prime} b^{\prime} b\right)=f \circ m\left(a a^{\prime}, b^{\prime} b\right)=f \circ m\left((a, \mathbf{1})\left(a^{\prime}, b^{\prime}\right)(\mathbf{1}, b)\right) \\
& =f \circ m(a, \mathbf{1})+f \circ m\left(a^{\prime}, b^{\prime}\right)+f \circ m(\mathbf{1}, b) \\
& =f(a)+f(g)+f(b) .
\end{aligned}
$$

Therefore $f$ is an adapted function. Now let $f$ be adapted and $(a, b),\left(a^{\prime}, b^{\prime}\right)$ 
$\in A \times B$. We compute that

$$
\begin{aligned}
f \circ m & \left((a, b)\left(a^{\prime}, b^{\prime}\right)\right)=f\left(a a^{\prime} b b^{\prime}\right)=f(a)+f\left(a^{\prime} b\right)+f(b) \\
& =f(a)+f\left(a^{\prime}\right)+f(b)+f\left(b^{\prime}\right)=f(a b)+f\left(a^{\prime} b^{\prime}\right) \\
& =f \circ m(a, b)+f \circ m\left(a^{\prime}, b^{\prime}\right) .
\end{aligned}
$$

So the function $f \circ m$ must be a homomorphism.

Lemma IV.10. Suppose that $G=A B$ is a product decomposition and the function $f: G \rightarrow \mathbf{R}$ is adapted. Then we have

$$
f^{\prime}(g)=f^{\prime}(\mathbf{1}) \circ \operatorname{Ad}(b) \text { for } g=a b \in G .
$$

Proof. For $h \in G$ we know that

$$
f \circ \lambda_{g}(h)=f(g h)=f(a b h)=f\left(a I_{b}(h) b\right)=f(a)+f \circ I_{b}(h)+f(b) .
$$

This implies that $f^{\prime}(g)=d f(g) \circ d \lambda_{g}(\mathbf{1})=d f(\mathbf{1}) \circ d I_{b}(\mathbf{1})=d f(\mathbf{1}) \circ \operatorname{Ad}(b)$.

Proposition IV.11. Suppose that $G=A B$ is a product decomposition and $\omega \in$ $([\mathbf{L}(A), \mathbf{L}(A)] \oplus[\mathbf{L}(B), \mathbf{L}(B)])^{\perp} \subseteq \widehat{\mathbf{L}(G)}$ is a functional such that the restrictions $\omega_{A}:=\left.\omega\right|_{\mathbf{L}(A)}$ and $\omega_{B}:=\left.\omega\right|_{\mathbf{L}(B)}$ satisfy the exactness condition of Lemma IV.6.2. Then we find a unique adapted function $f: G \rightarrow \mathbf{R}$ with $f^{\prime}(\mathbf{1})=\omega$.

Proof. The uniqueness follows from the fact that the morphism $f \circ m: A \times B \rightarrow$ $\mathbf{R}$ of connected Lie groups is completely determined by its left-invariant differential. It remains to prove the existence of $f$. The functional $\omega_{A}$ vanishes on the commutator algebra of $A$. Using Lemma IV.6 we find that the associated left-invariant 1 -form on $A$ is closed. Again with Lemma IV.6 and the assumptions from above we find a homomorphisms $f_{A}: A \rightarrow \mathbf{R}$ such that $f_{A}^{\prime}(\mathbf{1})=\omega_{A}$. A similar argument gives $f_{B}: B \rightarrow \mathbf{R}$ with $f_{B}^{\prime}(\mathbf{1})=\omega_{B}$. We set $f(a, b):=f_{A}(a)+f_{B}(b)$. Then $\tilde{f}: A \times B \rightarrow \mathbf{R}$ is a homomorphism of Lie groups with $d \tilde{f}(\mathbf{1})=\omega_{A}+\omega_{B}=\omega$. This shows that $f:=\tilde{f} \circ m^{-1}$ is the desired function.

\section{REFERENCES}

1. N. Bourbaki, Groupes et algèbres de Lie, Chapitre III, Hermann, Paris, 1971.

2. J. Dieudonné, Grundzüge der modernen Analysis, Band 3, Vieweg, 1976.

3. J. Faraut, Algèbres de Volterra et transformation de Laplace sphérique sur certains espaces symmétrique ordonnés, Sympos. Math. 29 (1987), 183-196.

4. G. B. Folland, Harmonic analysis in phase space, Princeton Univ. Press, Princeton, N.J., 1989.

5. S. W. Hawking and G. F. R. Ellis, The large scale structure of space-time, Cambridge Univ. Press, Cambridge, 1973.

6. S. Helgason, Differential geometry, Lie groups, and symmetric spaces, Academic Press, London, 1978.

7. J. Hilgert, K. H. Hofmann, and J. D. Lawson, Lie groups, convex cones, and semigroups, Oxford Univ. Press, 1989.

8. G. Hochschild, The structure of Lie groups, Holden-Day, San Francisco, Calif., 1965.

9. K. H. Hofmann, J. D. Lawson, and J. S. Pym (eds.), The analytic and topological theory of semigroups-trends and developments, De Gruyter, 1990.

10. J. D. Lawson, Maximal subsemigroups of Lie groups that are total, Proc. Edinburgh Math. Soc. 87 (1987), 497-501. 
11. G. I. Ol'shanskii, Invariant cones in Lie algebras, Lie semigroups, and the holomorphic discrete series, Funct. Anal. Appl. 15 (1982), 275-285.

12. _ Invariant orderings in simple Lie groups. The solution to E. B. Vinberg's problem, Funct. Anal. Appl. 16 (1982), 311-313.

Fachbereich Mathematik, Technische Hochschule Darmstadt, Schlossgartenstrasse 7, D-6100 DarmstadT, Germany 